\author{
Series A
}

I. MATHEMATICA

521

\title{
DARSTELLUNG VON CLIFFORDBÜNDELN
}

VON

GUIDO KARRER

H EL I N K I 1973

SUOM ALAINEN TIEDEAKA T E I A 
Copyright (C) 1973 by

Academia Scientiarum Fennica

ISBN 951-41-0083-2

Am 11. Februar 1972 vorgelegt von R. Nevisusxa

KESKUSKIPJAPAINO

HELSINKI 1973 


\section{Einleitung}

In den folgenden Ausführungen beschäftigen wir uns mit quadratischen Formen auf reellen und komplexen differenzierbaren Vektorbündeln, mit ihren Cliffordbündeln sowie deren Darstellungen.

Es ist hier unser Ziel, etwas ausführlicher als üblich auf die Eigenschaften einzugehen, die die Existenz von Differentialoperatoren (kovariante Ableitung, Diracoperator) und adjungierten Hermiteschen Formen auf diesen Strukturen versichern. Die Berechtigung für eine eher »elementare« Behandlung der Darstellungstheorie der Cliffordbündel scheint uns durch die Tatsache gegeben, daß in ihr die Ansätze und oft die wichtigsten Beispiele für verschiedene spezielle Thematas vorhanden sind: Brauergruppen von topologischen Räumen [5], Hermitesche Formen über Involutionsringen [7], elliptische Differentialoperatoren [1].

Thematisch ist die Arbeit in folgende Teile gegliedert: quadratische Formen und Cliffordalgebra, Darstellungen des Cliffordbündels, Diracoperator und adjungierte Hermitesche Formen, Beispiele.

\section{2. Äußere und Cliffordalgebra}

2.1. Konventionen. Unter einem Vektorraum verstehen wir einen endlichdimensionalen Vektorraum über den reellen oder komplexen Zahlen. Diese beiden Koeffizientenkörper werden wie üblich mit $\boldsymbol{R}$ (reell) bezw. $C$ (komplex) bezeichnet.

Unter einer quadratischen Form $Q$ auf einem Vektorraum $V$ verstehen wir, sofern nichts anderes vermerkt ist, eine nichtentartete quadratische Form auf $V$ mit Werten im Koeffizientenbereich von $V$. Das Paar $(V, Q)$ heiße ein Euklidischer Raum.

Der Index von $Q$ ist die Anzahl der negativen Eigenwerte der zu $Q$ assoziierten Bilinearform $B$ auf $V$ (falls $V$ ein reeller Vektorraum ist).

Der Term »Mannigfaltigkeit» bedeutet im folgenden, sofern nichts anderes vermerkt wird: reelle $C^{\infty}$-Mannigfaltigkeit von endlicher Dimension. Das Wort »differenzierbar« ist immer im Sinne von $C^{\infty}$ zu verstehen.

Ein Vektorbündel $\xi$ ist ein differenzierbares Vektorbündel über einer parakompakten Basismannigfaltigkeit $M$. Die Faser von $x \in M$ über dem 
Punkt $x \in M$ wird mit $\xi_{x}$ bezeichnet. Eine Bündelabbildung $\varphi: \xi \rightarrow \eta$, wo $\xi$ und $\eta$ Vektorbündel über demselben Koeffizientenbereich und derselben Basismannigfaltigkeit $M$ sind, ist eine differenzierbare Abbildung der Bündelräume, die für jedes $x \in M$ lineare Abbildungen $\varphi_{x}: \xi_{x} \rightarrow \eta_{x}$ induziert.

Ein Algebrabündel $\eta$ über der Basismannigfaltigkeit $M$ ist ein differenzierbares Faserbündel über $M$ mit typischer Faser $A$ und Strukturgruppe Aut $A$, wo $A$ eine assoziative, unitäre Algebra mit Koeffizienten aus $\boldsymbol{R}$ oder $C$ und Aut $A$ die Automorphismengruppe von $A$ ist.

Es sei $\xi$ ein Vektorbündel über der Basis $M$. Ist für jedes $x \in M$ die Faser $\xi_{x}$ Träger einer quadratischen Form $Q_{x}$, und hängt das Feld $Q=\left\{Q_{x}\right\}$ differenzierbar von $x$ ab, dann sprechen wir von einer quadratischen Form $Q$ auf $\xi$.

Ein $Q$-Bündel $(\xi, Q)$ ist dann ein Paar bestehend aus einem Vektorbündel $\xi$ und einer quadratischen Form $Q$ auf $\xi$.

Bei einem reellen $Q$-Bündel $(\xi, Q)$ über einer zusammenhängenden Basis $M$ haben alle induzierten quadratischen Formen $Q_{x}$ denselben Index. Man kann also in diesem Fall vom Index von $(\xi, Q)$ sprechen und entsprechend eine Klassifizierung der reellen $Q$-Bündel über $M$ in positiv- bzw. negativ-definite und indefinite vornehmen.

2.2. Reelle $Q$-Bündel. Wir wollen, ohne sie zu beweisen, auf einige Eigenschaften reeller $Q$-Bündel hinweisen, die in genauer Analogie zu bekannten Eigenschaften reeller Euklidischer Räume stehen. Dabei betrachten wir nur Vektorbündel über ein und derselben Basismannigfaltigkeit $M$ und setzen voraus, $M$ sei zusammenhängend.

Zwei reelle $Q$-Bündel $(\xi, Q)$ und $\left(\xi^{\prime}, Q^{\prime}\right)$ heissen isomorph, falls es eine Bündelabbildung $\varphi: \xi \rightarrow \xi^{\prime}$ mit der für alle Fasern gültigen Eigenschaft

$$
Q_{x}^{\prime}\left(\varphi_{x} v_{x}\right)=Q_{x}\left(v_{x}\right) \quad \text { für alle } v_{x} \in \xi_{x}, \quad \operatorname{dim} \xi_{x}=\operatorname{dim} \xi_{x}^{\prime},
$$

gibt. Da die quadratischen Formen als nichtentartet vorausgesetzt sind, so ist eine solche Bündelabbildung ein Isomorphismus der Vektorbündel. Es gilt nun:

2.2.1. Zwei positiv-definite reelle $Q$-Bündel $(\xi, Q)$ nnd $\left(\xi^{\prime}, Q^{\prime}\right)$ sind genau dann isomorph, wenn die Vektorbündel $\xi$ und $\xi^{\prime}$ isomorph sind.

Dasselbe gilt natürlich auch, wenn beide $Q$-Bündel negativ-definit siud.

Um die entsprechende Aussage für indefinite $Q$-Bündel formulieren zu können wird der Begriff der »direkten Summe» eingeführt. Unter der direkten Summe zweier (reellen oder komplexer) $Q$-Bündel $(\xi, Q)$ und $\left(\xi^{\prime}, Q^{\prime}\right)$ wird das Paar $\left(\xi \oplus \xi^{\prime}, Q \oplus Q^{\prime}\right)$ verstanden, wo $\xi \oplus \xi^{\prime}$ die WhitneySumme der Vektorbündel und $Q^{\prime \prime}=Q \oplus Q^{\prime}$ die quadratische Form 


$$
Q_{x}^{\prime \prime}\left(v_{x}, v_{x}^{\prime}\right)=Q_{x}\left(v_{x}\right)+Q_{x}^{\prime}\left(v_{x}^{\prime}\right)
$$

auf $\xi_{x} \oplus \xi_{x}^{\prime}$ ist. Mit Hilfe einer positiv-definiten quadratischen Form auf $\xi$ beweist man dann:

2.2.2. Jedes reelle $Q$-Bündel $(\xi, Q)$ ist direkte Summe zweier definiter reeller $Q$-Bündel $\left(\xi_{+}, Q_{+}\right)$und $\left(\xi_{-}, Q_{-}\right)$, wobei da.s erste positiv-, das zweite negativ-definit ist, und $\operatorname{dim} \xi_{-}=t=\operatorname{ind} Q$ ist.

Die Verallgemeinerung von 2.2.1 lautet nun:

2.2.3. $(\xi, Q)$ und $\left(\xi^{\prime}, Q^{\prime}\right)$ seien reelle $Q$-Bündel mit den nach 2.2.2 existierenden definiten Zerlegungen

$$
\begin{aligned}
& (\xi, Q)=\left(\xi_{+}, Q_{+}\right) \oplus\left(\xi_{-}, Q_{-}\right), \\
& \left(\xi^{\prime}, Q^{\prime}\right)=\left(\xi_{+}^{\prime}, Q_{+}^{\prime}\right) \oplus\left(\xi_{-}^{\prime}, Q_{-}^{\prime}\right) .
\end{aligned}
$$

Sie sind genau dann isomorph, wenn es Vektorbündelisomorphismen $\xi_{+} \rightarrow \xi_{+}^{\prime}$ und $\xi_{-} \rightarrow \xi_{-}^{\prime}$ gibt.

2.3. Komplexe $Q$-Bündel. Die Operation der komplexen Erweiterung führt ein reelles Vektorbündel $\xi$ in ein komplexes Tektorbündel $\boldsymbol{\xi} \otimes \boldsymbol{C}$, und eine auf $\xi$ definierte quadratische Form $Q$ in eine auf $\xi \otimes C$ definierte quadratische Form $Q \otimes C$ über. Das auf diese Weise aus dem reellen $Q$-Bündel $(\xi, Q)$ erhaltene komplexe $Q$-Bündel sei mit $(\xi, Q) \otimes C$ bezeichnet.

2.3.1. $Q$ und $Q^{\prime}$ seien quadratische Formen auf einem reellen Vektorbündel $\xi$. Dann sind die komplexen Erweiterungen $(\xi, Q) \otimes C$ und $\left(\xi, Q^{\prime}\right) \otimes C$ isomorph.

Beweis. Man hat 2.3.1 offensichtlich nur für den Fall zu beweisen, daß eine der beiden quadratischen Formen, etwa $Q$, positiv-definit ist. Es sei dann

$$
\left(\xi, Q^{\prime}\right)=\left(\xi_{+}, Q_{+}^{\prime}\right) \oplus\left(\xi_{-}, Q_{-}^{\prime}\right)
$$

die in 2.2.2 gegebene definite Zerlegung. Dann jst das reelle $Q$-Bündel

$$
\left(\xi, Q_{1}\right)=\left(\xi_{+}, Q_{+}^{\prime}\right) \oplus\left(\xi_{-},-Q_{-}^{\prime}\right)
$$

positiv-definit, somit gemäß 2.2.1 isomorph $(\xi, Q)$, und daraus folgt, daß auch die komplexen Erweiterungen $(\xi, Q) \otimes C$ und $\left(\xi, Q_{1}\right) \otimes C$ isomorph sind. Schließlich bleibt 2.3.1 nur noch unter der Voraussetzung $Q^{\prime}=-Q$ zu zeigen. In diesem Fall ist aber die Multiplikation mit $i$ der gewünschte Isomorphismus von $(\xi, Q) \otimes \boldsymbol{C}$ auf $\left(\xi, Q^{\prime}\right) \otimes \boldsymbol{C}$.

Ist $(\xi, Q)$ ein reelles $Q$-Bündel, dann erhält man daraus durch komplexe Erweiterung ein komplexes $Q$-Bündel. Es soll noch gezeigt werden, daß auch die Umkehrung dieses Sachverhaltes richtig ist. 
2.3.2. Jedes komplexe $Q$-Bündel wird durch Komplexifizierung aus einem reellen $Q$-Bündel erhalten.

Beachtet man zudem noch, daß jedes reelle Vektorbündel über einer parakompakten Basismannigfaltigkeit $M$ eine positiv-definite quadratische Form trägt, so erhält man die folgende Aussage als

Korollar. Ein komplexes Vektorbündel trägt genau dann eine quadratische Form, wenn es durch Komplexifizierung aus einem reellen Vektorbündel entsteht.

Beweis von 2.3.2. $\left(\xi_{C}, Q_{C}\right)$ sei ein komplexes $Q$-Bündel. Wir benützen folgende Bezeichnungen:

$$
\begin{aligned}
\xi_{R} & =\xi_{C} \text { als reelles Vektorbündel aufgefasst; } \\
J & =\text { Multiplikation mit } i \text { in } \xi_{C} \\
Q_{1} & =\operatorname{Re} Q_{C} \quad(\operatorname{Re}=\text { Realteil }) \\
Q_{2} & =\operatorname{Im} Q_{C} \quad(\operatorname{Im}=\text { Imaginärteil })
\end{aligned}
$$

Weiter sei $Q^{\prime}$ eine positiv-definite quadratische Form auf $\xi_{R}$. Dann ist die quadratische Form $Q$ auf $\xi_{R}$, definiert durch

$$
Q_{x}\left(v_{x}\right)=Q_{x}^{\prime}\left(v_{x}\right)+Q_{x}^{\prime}\left(i v_{x}\right), \quad v_{x} \in \xi_{R x}
$$

wieder positiv-definit, und zudem ist $J$ eine in $Q$ orthogonale Abbildung.

Es seien nun $B_{1}, B_{2}, B$ die zu $Q_{1}, Q_{2}, Q$ assoziierten Bilinearformen. Dann gibt es Vektorbündelisomorphismen $\varphi_{1}, \varphi_{2}: \xi_{R} \rightarrow \xi_{R}$ derart, daß auf der Faser $\xi_{\boldsymbol{R} x}$ gilt:

$B_{1 x}\left(v_{x}, v_{x}^{\prime}\right)=B_{x}\left(\varphi_{1 x} v_{x}, v_{x}^{\prime}\right), \quad B_{2 x}\left(v_{x}, v_{x}^{\prime}\right)=B_{x}\left(\varphi_{2 x} v_{x}, v_{x}^{\prime}\right) ; \quad v_{x}, v_{x}^{\prime} \in \xi_{R x}$. $\varphi_{1}$ und $\varphi_{2}$ sind in der positiv-definiten Form $B$ selbstadjungiert. $\xi_{\boldsymbol{R}}$ wird somit in zwei Bündel $\xi_{+}$und $\xi_{-}$zerlegt

$$
\xi_{R}=\xi_{+} \oplus \xi_{-},
$$

wobei die Faser $\xi_{+x}$ von den Eigenvektoren von $\varphi_{1 x}$ mit positivem, $\xi_{-x}$ von denjenigen mit negativem Eigenwert aufgespannt wird. Die Restriktion von $Q_{1}$ auf $\xi_{+}$ist positiv-, auf $\xi_{-}$negativ-definit. Außerdem sind $\xi_{+}$und $\xi_{-}$sowohl in $B$ als auch in $B_{1}$ orthogonal. Aus diesen Beziehungen ergeben sich die folgenden Gleichungen

$$
\begin{aligned}
J^{-1} \varphi_{1} J & =-\varphi_{1} ; \\
\varphi_{2} & =-\varphi_{1} J .
\end{aligned}
$$

Die erste dieser Gleichungen impliziert $\xi_{-}=J\left(\xi_{+}\right)$, also

$$
\xi_{R}=\xi_{+} \oplus J\left(\xi_{+}\right) .
$$


Somit ist $\xi_{C}$ das aus dem reellen Bündel $\xi_{+}$komplexifizierte Bündel: $\xi_{C}=\xi_{+} \otimes C$. Aus der zweiten Gleichung folgt für $v_{x} \in \xi_{+x}$

$$
Q_{2 x}\left(v_{x}\right)=B_{x}\left(\varphi_{2 x} v_{x}, v_{x}\right)=-B_{x}\left(\varphi_{1 x}\left(i v_{x}\right), v_{x}\right)=0,
$$

also ist die Restriktion von $Q_{C}$ auf $\xi_{+}$reellwertig und gleich der Restriktion von $Q_{1}$ auf $\xi_{+}$, die mit $Q_{1}^{+}$bezeichnet sei.

Die komplexe Erweiterung von $Q_{1}^{+}$auf $\xi_{C}$ stimmt auf $\xi_{+}$mit $Q_{C}$ überein. Also ist $Q_{C}=Q_{1}^{+} \otimes C$.

2.4. Definition des Cliffordbündels. Für die Definition und Eigenschaften von Cliffordalgebren wird prinzipiell auf [3] verwiesen. Ist $(V, Q)$ ein Euklidischer Raum (vgl. 2.1), dann wird mit $C(Q)$ die Cliffordalgebra von $(V, Q)$ bezeichnet.

Definition. $(\xi, Q)$ sei ein $Q$-Bündel mit Basismannigfaltigkeit $M$. Ein Algebrabündel $\gamma$ über $M$ mit der für die Fasern gültigen Eigenschaft

$$
\gamma_{x}=C\left(Q_{x}\right), \quad x \in M,
$$

ist ein Cliffordbündel von $(\xi, Q)$.

Aus der Eindeutigkeit der Cliffordalgebra folgt, daß alle Cliffordbündel von $(\xi, Q)$ untereinander isomorph sind. Wir wählen eines von diesen Cliffordbündeln aus und bezeichnen es als das Clifford̈bündel von $(\xi, Q)$.

Die Universalitätseigenschaft der Cliffordalgebra ([3], II.1.1, S. 39) überträgt sich auf das Cliffordbündel $\gamma$ von $(\xi, Q)$ :

2.4.1. Es sei $\eta$ ein reelles bzw. komplexes Algebrabündel über $M$, $\varphi: \xi \rightarrow \eta$ eine Bündelabbildung mit der für jedes $x \in M$ gültigen Eigenschaft

$$
\left(\varphi_{x} v_{x}\right)^{2}=Q_{x}\left(v_{x}\right) 1_{x}
$$

$v_{x} \in \xi_{x}$ und $\mathrm{1}_{x}=$ Einselement von $\eta_{x}$. Dann gibt es eine eindeutig bestimmte Abbildung der Algebrabündel, $\tilde{\varphi}: \gamma \rightarrow \eta$, die $\varphi$ erweitert.

Dabei haben wir angenommen, $\xi$ sei ein Teilbündel von $\gamma$. Daß diese Annahme berechtigt ist, folgt aus der entsprechenden Eigenschaft der Cliffordalgebren. Für $v_{x} \in \xi_{x}$ gilt die Produkteigenschaft

$$
\left(v_{x}\right)^{2}=Q_{x}\left(v_{x}\right) 1_{x}, \quad 1_{x}=\text { Einselement von } \gamma_{x} ;
$$

dies ergibt zusammen mit 2.2.1 und 2.4.1 den Satz

2.4.2. Sind $(\xi, Q)$ und $\left(\xi, Q^{\prime}\right)$ reelle $Q$-Bündel über $M$, beide entweder positiv- oder negativ-definit, dann sind ihre Cliffordbündel isomorph.

Ein reelles $Q$-Bündel $(\xi, Q)$ geht durch Komplexifizierung in ein komplexes $Q$-Bündel $(\xi, Q) \otimes C$ über. Sind $\gamma, \gamma^{\prime}$ die Cliffordbündel von $(\xi, Q)$ bzw. $(\xi, Q) \otimes C$, sowie $\gamma \otimes C$ die Komplexifizierung von $\gamma$, dann folgt aus dem entsprechenden Satz über Cliffordalgebren ([3], 
A. I. 521

II.1.5, S. 41), daß $\gamma^{\prime}=\gamma \otimes C$. Wir nennen $\gamma^{\prime}$ das komplexe Cliffordbündel des reellen $Q$-Bündels $(\xi, Q)$.

Aus 2.3.1 und 2.4.1 gewinnt man nun folgendes Resultat:

2.4.3. $Q$ und $Q^{\prime}$ seien beliebige (nicht-entartete) quadratische Formen auf dem reellen Vektorbündel $\xi$. Dann sind die zu $(\xi, Q)$ und $\left(\xi, Q^{\prime}\right)$ gehörenden komplexen Cliffordbündel isomorph.

2.5. Existenz des Cliffordbündels. $G$ sei eine Liegruppe und $V$ ein endlichdimensionaler Vektorraum, auf dem $G$ differenzierbar operiert, d.h. es sei $V$ ein $G$-Modul. Man kann bekanntlich (siehe etwa [9], 3.2.d, S. 45) jedem $G$-Prinzipalbündel $\tilde{\xi}$ über der Basismannigfaltigkeit $M$ ein Vektorbündel über $M$ mit $V$ als typischer Faser zuordnen, das im folgenden mit $\tilde{\xi} \otimes_{G} V$ bezeichnet sei. Ist $V^{\prime}$ Teilmodul des $G$-Moduls $V$, dann kann man $\tilde{\xi} \otimes_{G} V^{\prime}$ als Teilbündel von $\tilde{\xi} \otimes_{G} V$ auffassen.

Es sei nun $(\xi, Q)$ ein $G$-Bündel über $M, V$ die typische Faser von $\xi, Q_{0}$ eine quadratische Form auf $V$ mit (für reelles $\xi$ ) gleichem Index wie $Q, O$ die orthogonale Gruppe von $\left(V, Q_{0}\right)$ und $C$ die Cliffordalgebra von $\left(V, Q_{0}\right)$. Erstens bestimmt $(\xi, Q)$ ein $O$-Prinzipalbündel $\tilde{\xi}$ über $M$ derart, daß $\tilde{\xi} \otimes_{o} V=\xi$. Aus der Universalitätseigenschaft [3], II.1.1, S. 39) der Cliffordalgebra $C$ folgt weiter, daß $O$ auch auf $C$ operiert, und zwar als eine Automorphismengruppe von $C$. Denn jedes $g \in O$ läßt sich eindeutig zu einem Automorphismus $\tilde{g}$ von $C$ erweitern. Das zu $\tilde{\xi}$ assoziierte Bündel $\tilde{\xi} \otimes_{0} C$ ist dann, wie man sofort einsieht, das Cliffordbündel $\gamma$ von $(\xi, Q)$.

Da $\gamma$ definitionsgemäß überhaupt nur bis auf Isomorphie durch $(\xi, Q)$ festgelegt ist, so ist es ganz zweckmäßig, die Beziehung zwischen $(\xi, Q)$ und $\gamma$ durch ihre Isomorphieklassen auszudrücken. Dabei verweisen wir auf den in 2.2 eingeführten Isomorphiebegriff. Die Isomorphieklasse von $(\xi, Q)$ ist in der Terminologie von [9], 3.2.a, S. 44, ein $O$-Bündel über $M$. Ebenso: Ist Aut $C$ die Automorphismengruppe der Algebra $C$, dann ist die Isomorphieklasse von $\gamma$ ein Aut $C$-Bündel über $M$.

Es sei nun $\varepsilon: O \rightarrow$ Aut $C$ der oben erwähnte Erweiterungshomomorphismus. Da (für eine Liegruppe $G$ ) die $G$-Bündel über $M$ Elemente der Kohomologiemenge $H^{1}(M, G)$ sind (siehe [9], S. 42), so defifiniert $\varepsilon$ eine Abbildung $\varepsilon_{*}: H^{1}(M, O) \rightarrow H^{1}(M$, Aut $C)$, und die Isomorphieklasse von $\gamma$ ist das $\varepsilon_{*}$-Bild derjenigen von $(\xi, Q)$ :

$$
\varepsilon_{*}((\xi, Q))=(\gamma) .
$$

2.6. Beziehung zwischen äußerem und Cliffordbündel. Ist $\xi$ ein Vektorbündel über der Basismannigfaltigkeit $M$, dann bezeichnet 
$\wedge \xi$ das äußere Bündel von $\xi$. Für jedes $x \in M$ ist also die Faser $(\wedge \xi)_{x}$ die äußere Algebra von $\xi_{x}$.

Es sei weiter $Q$ eine quadratische Form auf $\xi$ und $\gamma$ das Cliffordbündel von $(\xi, Q)$. Dann gilt:

2.6.1. Es gibt einen kanonischen Vektorbündelisomorphismus $\mu: \gamma \rightarrow \wedge \xi$.

Allerdings respektiert $\mu$ nicht die multiplikative Struktur der Algebrabündel $\gamma$ und $\wedge \xi$.

Wir wollen im folgenden diesen Isomorphismus konstruieren. Es handelt sich dabei lediglich um eine algebraische Aufgabe, die darin besteht, für jedes $x \in M$ einen kanonischen linearen Isomorphismus $\mu_{x}$ : $C_{x} \rightarrow \wedge \xi_{x}$ nachzuweisen (wie dies z.B. in [3], II.2.1, S. 38 gemacht wird). Da die Isomorphismen $\mu_{x}$ kanonisch definiert sind, werden sie sich dann zu einer differenzierbaren Bündelabbildung zusammensetzen.

$\mathrm{Um}$ das algebraische Problem zu fixieren, sei $(V, Q)$ ein Euklidischer Raum, $C$ die Cliffordalgebra von $(V, Q), E$ die äußere Algebra von $V$, und schließlich $B$ die zu $Q$ assoziierte Bilinearform. Wir können annehmen, daß $V \subset C, V \subset E$, und außerdem, daß $C$ und $E$ ein gemeinsames Einselement 1 besitzen.

Für festes $v \in V$ existieren Antiderivationen in $C$ und $E$, beide mit dem gleichen Symbol $\partial_{v}$ bezeichnet $\left(\partial_{v}=\delta_{v}\right.$ in der Bezeichnung von [3], II.2.1), sodaß für $w \in V$ gilt

$$
\partial_{v} w=B(v, w) 1 .
$$

Es sei $L_{v}$ die Linksmultiplikation mit $v \in V$ in $E$. Dann wird durch

$$
v \mapsto L_{v}+\partial_{r}
$$

eine lineare Abbildung $v: V \rightarrow$ End $E$ (= Endomorphismenalgebra des Vektorraumes $E$ ) erklärt. Man berechnet nun sofort

$$
\left(L_{v}+\partial_{v}\right)^{2}=Q(v) \mathrm{id}_{E} .
$$

Gemäß der Universalitätseigenschaft von $C$ ([3], II.1.1, S. 39) lässt sich $v$ zu einer Darstellung $\tilde{\mu}: C \rightarrow$ End $E$ erweitern, und man gewinnt daraus eine lineare Abbildung $\mu: C \rightarrow E$, indem man den Endomorphismus $\tilde{\mu} c$ auf $1 \in E$ anwendet:

$$
\mu c:=(\tilde{\mu} c) 1 \text {. }
$$

Man zeigt sehr leicht, daß $\mu$ eine Inverse besitzt. Ist nämlich $L_{v}^{\prime}$ die Linksmultiplikation mit $v \in V$ in $C$, dann wird $\mu^{-1}$ auf analoge Weise wie vorhin durch die Abbildung $v \rightarrow L_{v}^{\prime}-\partial_{v}$ erzeugt. Aus der Dəfinition von $\mu$ erhält man sofort die folgenden Eigenschaften:

2.6.2. $\mu v=v$ für $v \in V$ und $\mu 1=1$. Ist $\left\{v_{i}\right\}, i=1, \ldots, p$, ein 
System paarweise in $B$ orthogonaler Vektoren von $V$, dann ist $\mu\left(v_{1} \ldots v_{p}\right)$ $=v_{1} \wedge \ldots \wedge v_{p}$. Dabei ist $v_{1} \ldots v_{p}$ das Produkt in $C, v_{1} \wedge \ldots \wedge v_{p}$ dasjenige in $E$.

2.7. Der Schnitt $c_{\Omega}$. Vorerst soll wieder eine algebraische Betrachtung gemacht werden. Es sei $(V, Q)$ ein reeller Euklidischer Raum und $\Omega$ eine Orientierung von $V$. Dann gilt für zwei in $\Omega$ positiv orientierte und in $B$ orthonormierte Basen $\left\{v_{i}\right\}$ und $\left\{v_{i}^{\prime}\right\}, i=1, \ldots, n=\operatorname{dim} V$, von $V$ :

$$
v_{1} \wedge \ldots \wedge v_{n}=v_{i}^{\prime} \wedge \ldots \wedge v_{n}^{\prime}
$$

Das Volumelement ist dann $e_{\Omega}=v_{1} \wedge \ldots \wedge v_{n}$, und wir setzen

$$
c_{\Omega}:=\mu^{-1} e_{\Omega} \in C .
$$

$c_{\Omega}$ ist damit durch die Orientierung $\Omega$ von $V$ festgelegt; wegen 2.6.2 kann es mittels einer in $\Omega$ positiv orientierten und in $B$ orthonormierten Basis $\left\{v_{i}\right\}$ von $V$ durch das Produkt in $C$

$$
c=v_{1} \ldots v_{n}
$$

dargestellt werden. Nun gilt für eine solche Basis die Antikommutationsregel (in $C$ ) (siehe [3], (1), S. 39)

$$
v_{i} v_{k}+v_{k} v_{i}= \pm 2 \delta_{i k} 1 \text {. }
$$

Man erhält daraus die folgenden Eigenschaften von $c_{\Omega}$ :

2.7.1. Die ganze Zahl $m$ sei so gewählt, daß entweder $\operatorname{dim} V=2 \mathrm{~m}$ oder $=2 m+1$ ist, und es sei $t$ der Index von $Q$. Dann ist $\left(c_{\Omega}\right)^{2}=(-1)^{m+t}$. Ist $\operatorname{dim} V$ ungerade, so ist $v c_{\Omega}=c_{\Omega} v$ für $v \in V$. Ist $\operatorname{dim} V$ gerade, so ist $v c_{\Omega}=-c_{\Omega} v$ für $v \in V$, und zu jedem $c \in C$ mit der Eigenschaft $v c=-c v$ für $v \in V$ gibt es ein solches $\lambda \in R$, daß $c=\lambda c_{\Omega}$ ist.

Die letzte Eigenschaft von $c_{\Omega}$ folgt daraus, daß $C$ für gerade Dimensionen von $V$ eine zentrale Algebra ist ([3], II.2.1, S. 42).

Diese algebraischen Definitionen lassen sich nun alle faserweise auf ein reelles orientiertes $Q$-Bündel $(\xi, Q)$ übertragen. Man erhält also insbesondere einen (differenzierbaren) Schnitt $c_{\Omega}$ in $\gamma$, dem Cliffordbündel von $(\xi, Q)$, dessen Wert $c_{\Omega x}$ in $x \in M$ die Eigenschaften 2.7.1 hat.

2.8. Der *-Operator. $(V, Q)$ sei ein reeller Euklidischer Raum, $E$ die äußere Algebra von $V, e_{\Omega}$ das durch $Q$ und eine Orientierung $\Omega$ von $V$ festgelegte Volumelement.

Die in 2.6 definierte Antiderivation $\partial_{v}$ in $E$ hat die Eigenschaft $\left(\partial_{r}\right)^{2}=0$. Man kann also die Abbildung $\tau: v \rightarrow \partial_{v}$ zu einem Antihomo- 
morphismus $\tau: E \rightarrow$ End $E$ fortsetzen. Das »Anti» bezieht sich dabei auf die Produkteigenschaft

$$
\tilde{\tau}\left(e \wedge e^{\prime}\right)=\left(\tilde{\tau} e^{\prime}\right)(\tilde{\tau} e), \quad e, e^{\prime} \in E .
$$

Durch

$$
* e:=(\tilde{\tau} e) e_{\Omega}
$$

gewinnt man daraus eine lireare Abbildung $*: E \rightarrow E$. Überträgt man sie faserweise auf das äußere Bündel eines reellen orientierten $Q$-Bündels, so erhält man den de-Rhamschen *-Operator ([11], § 24, S. 121).

2.9. Linearer Zusammenhang. Es sei $M$ die Basismannigfaltigkeit der betrachteten Vektorbündel, $\mathcal{F}_{\boldsymbol{R}}\left(\mathcal{F}_{\boldsymbol{C}}\right)$ der Ring der differenzierbaren reell- (komplex-)wertigen Funktionen auf $M$. Der Schnittmodul $\Gamma \xi$ eines Vektorbündels $\xi$ über $M$ wird als $\bar{F}_{\boldsymbol{R}}-\left(\mathcal{F}_{C^{-}}\right)$Modul aufgefaßt. Wird er als $\boldsymbol{R}$ - $\left(\boldsymbol{C}\right.$-) Vektorraum betrachtet, dann schreiben wir $\Gamma_{\boldsymbol{R}} \xi$ $\left(\Gamma_{C} \xi\right)$. Für das kontravariante Tangentialbündel $\tau(M)$ setzen wir $\mathscr{C}=\Gamma \tau(M)$, für das kovariante $\tau^{*}(M)$ entsprechend $\mathcal{C}^{*}=\Gamma \tau^{*}(M)$.

Es sei $\nabla$ ein linearer Zusammenhang auf dem Vektorbündel $\xi, \nabla_{h} \cdot s$ die kovariante Ableitung von $s \in \Gamma \xi$ in Richtung $h \in \mathcal{X}$. Bekanntlich gelten die folgenden Regeln:

a) Für festes $s \in \Gamma \xi$ ist die Abbildung

$$
\nabla \cdot s: \quad \oiiint \rightarrow \Gamma \xi, \quad h \mapsto \nabla_{h} \cdot s,
$$

ein Modulhomomorphismus $\mathscr{X} \rightarrow \Gamma \xi$.

b) Für festes $h \in \mathcal{C}$ ist die Abbildung

$$
s \mapsto \nabla_{h} \cdot s
$$

ein Vektorraumhomomorphismus $\Gamma_{\boldsymbol{R}} \xi \rightarrow \Gamma_{\boldsymbol{R}} \xi$ (bzw. $\Gamma_{\boldsymbol{C}} \xi \rightarrow \Gamma_{\boldsymbol{C}} \xi$ ), und es gilt

$$
\nabla_{h} \cdot(\lambda s)=\lambda\left(\nabla_{h} \cdot s\right)+(h \cdot \lambda) s,
$$

wo $h \cdot \lambda$ die Ableitung von $\lambda \in \mathcal{F}_{\boldsymbol{R}}\left(\in \bar{F}_{C}\right)$ in Richtung $h \in \mathcal{X}$ bedeutet.

Ist $\xi$ ein Algebrabündel, dann verlangen wir noch zusätzlich:

c) $\nabla_{h} \cdot\left(s_{1} s_{2}\right)=\left(\nabla_{h} \cdot s_{1}\right) s_{2}+s_{1}\left(\nabla_{h} \cdot s_{2}\right), s_{1} s_{2}=$ Produkt in $\Gamma \xi$.

Falls ein linearer Zusammenhang $\nabla$ und eine bilineare bzw. Hermitesche Form $H$ auf dem Vektorbündel $\xi$ die Ricci-Identität

$$
h \cdot H\left(s_{1}, s_{2}\right)-H\left(\nabla_{h} \cdot s_{1}, s_{2}\right)-H\left(s_{1}, \nabla_{h} \cdot s_{2}\right)=0
$$

erfüllen, heißt $\nabla$ ein linearer Zusammenhang auf dem Paar $(\xi, H)$. 
Ein linearer Zusammenhang auf dem $Q$-Bündel $(\xi, Q)$ ist ein solcher des Paares $(\xi, B)$, wo $B$ die zu $Q$ assoziierte Bilinearform ist.

Nun sei $\wedge \xi$ das äußere Bündel von $\xi$ und $\nabla$ ein linearer Zusammenhang auf $\xi$. Es gibt eine eindeutige Erweiterung $e_{e} \nabla$ von $\nabla$ auf $\wedge \xi$, die auf zerlegbaren Schnitten $s=v_{1} \wedge \ldots \wedge v_{r}, v_{i} \in \Gamma \xi$, die Werte

$$
\nabla_{h} \cdot s=\sum_{i=1}^{r} v_{1} \wedge \ldots \wedge\left(\nabla_{h} \cdot v_{i}\right) \wedge \ldots \wedge v_{r}
$$

annimmt. Ist $(\xi, Q)$ ein reelles $Q$-Bündel mit Cliffordbündel $\gamma$, dann wird die Erweiterung $e \nabla$ mittels des kanonischen Isomorphismus $\mu$ : $\gamma \rightarrow \wedge \xi \quad\left(\left(\right.\right.$ vgl. 2.6) in einen linearen Zusammenhang $\mu^{-1}{ }_{e} \nabla$ auf dem Vektorbündel $\gamma$ übertragen. Man verifiziert nun, daß $\mu^{-1}{ }_{e} \nabla$ ein linearer Zusammenhang auf dem Algebrabündel $\gamma$ ist, d.h. daß c) erfüllt ist, falls $\nabla$ ein linearer Zusammenhang auf $(\xi, Q)$ ist. $\mu^{-1}{ }_{e} \nabla$ ist dann die eindeutige Erweiterung von $\nabla$ auf $\gamma$.

Wir wollen die eindeutigen Erweiterungen eines linearen Zusammenhanges $\nabla$ auf $(\xi, Q)$ zu solchen auf $\gamma$ und $\wedge \xi$ wieder mit $\nabla$ bezeichnen. Ist $\Omega$ eine Orientierung von $\xi$, dann gilt bekanntlich

$$
\nabla \cdot e_{\Omega}=0
$$

( $e_{\Omega}=$ Volumelement, vgl. 2.7). Wegen $c_{\Omega}=\mu^{-1} e_{\Omega} \quad$ (vgl. 2.7) gilt also auch

$$
\nabla \cdot c_{\Omega}=0
$$

Schließlich sei noch auf eine Beziehung zwischen den Antiderivationen $\partial_{v}$ (vgl. 2.6) und $\nabla$ hingewiesen, wobei beide, $\partial_{v}$ und $\nabla$, entweder auf $\gamma$ oder auf $\wedge \xi$ operierend gedacht werden. Aus der Definition von $\partial_{v}$ folgt sofort

$$
\nabla_{h} \cdot\left(\partial_{v} s\right)-\partial_{v}\left(\nabla_{h} \cdot s\right)=\partial_{\Gamma_{h} \cdot v} s,
$$

wobei $v \in \Gamma \xi, h \in \mathcal{X}$ und $s \in \Gamma \wedge \xi$ (bzw. $s \in \Gamma \gamma$ ).

\section{S- und SC-Strukturen}

Viele der folgenden Betrachtungen gelten nur für orientierte reelle Vektorbündel. Wir treffen deshalb die Vereinbarung, daß in diesem Kapitel »reelles Vektorbündel" gleichbedeutend ist mit »orientiertes reelles Vektorbündel«. Außerdem sollen alle Faserbündel über ein und derselben parakompakten und zusammenhängenden Basismannigfaltigkeit $M$ definiert sein. 
3.1. $\alpha$-Bündel. Gegeben sei ein Algebrabündel $\alpha$. Unter einem $\alpha$-Bündel $\xi_{\Phi}$ verstehen wir ein Tripel $(\alpha, \xi, \Phi), \xi$ ein Vektorbündel und $\Phi$ ein Algebrahomomorphismus $\alpha \rightarrow$ End $\xi$ ( End $\xi=$ Bündel der Endomorphismen von $\xi$ ). Insbesondere soll $\Phi_{x}$, die Restriktion von $\Phi$ auf $\alpha_{x}$, das Einselement $1_{x}$ von $\alpha_{x}$ in das Einselement $\operatorname{id}_{\xi_{x}}$ von End $\xi_{x}$ überführen.

In einem $\alpha$-Bündel $\xi_{\Phi}$ ist jede Faser $\xi_{x}$ ein $\alpha_{x}$-Modul im algebraischen Sinn. Entsprechend hat man auch bei $\alpha$-Bündeln den Begriff der Isomorphie, der direkten Summe sowie des Tensorproduktes eines $\alpha$-Bündels mit einem Vektorbündel.

3.2. Definition der S-Strukturen. $\quad \gamma$ sei das Cliffordbündel eines reellen $Q$-Bündels $(\xi, Q)$. Unter einer S-Struktur auf $(\xi, Q)$ verstehen wir die Auszeichnung eines $\gamma$-Bündels $\eta_{\Phi}$. Das zugrundeliegende Vektorbündel $\eta$ wird als das Spinorbündel der $S$-Struktur $\eta_{\Phi}$ bezeichnet. Eine S-Struktur auf dem reellen $Q$-Bündel $(\xi, Q)$ kann auch durch folgende Daten gegeben werden:

S 1. Ein reelles oder komplexes Vektorbündel $\eta$ mit einer Bündelabbildung $\varphi: \xi \rightarrow$ End $\eta$, die die Eigenschaft

$$
\left(\varphi_{x} v_{x}\right)^{2}=\left(Q_{x}\left(v_{x}\right)\right) \mathrm{id}_{\eta_{x}}, \quad v_{x} \in \xi_{x}
$$

für alle Fasern $\xi_{x}$ von $\xi$ hat.

Denn in jeder S-Struktur $\eta_{\Phi}$ von $(\xi, Q)$ liefert die Restriktion des Homomorphismus $\Phi_{x}: \gamma_{x} \rightarrow$ End $\eta_{x}$ auf $\xi_{x}$ eine Abbildung $\varphi_{x}$ mit der Eigenschaft S 1, und umgekehrt kann jede solche wegen der Univeralitätseigenschaft 2.4.1 zu einem eindeutigen Homomorphismus $\Phi_{x}: \gamma_{x} \rightarrow$ End $\eta_{x}$ erweitert werden.

3.2.1. Ist $\operatorname{dim} \xi$ gerade $(=2 \mathrm{~m})$ und $t$ der Index von $Q$, dann isi das Spinorbündel $\eta$ einer S-Struktur $\eta_{\Phi}$ auf $(\xi, Q)$ direkte Summe zweier Vektorbündel $\eta_{+}$und $\eta_{-}$gleicher Dimension, falls entweder $\eta$ reell und. $m+t$ gerade oder $\eta$ komplex ist. $\eta_{\div}$und $\eta_{-}$sind die Halbspinorbündel von $\eta_{\Phi}$.

Der Beweis von 3.2.1 benützt die Existenz des Schnittes $c_{\Omega}$ in $\gamma$ (vgl. 2.7). Es sei $\Phi: \gamma \rightarrow$ End $\eta$ der Homomorphismus, der $\gamma$ auf $\eta$ operieren läßt. Dann ist $\Phi c_{\Omega}$ ein Vektorbündelisomorphismus von $\eta$ mit der Eigenschaft

$$
\left(\Phi c_{\Omega}\right)^{2}=(-1)^{m+t} \mathrm{id}_{\eta}
$$

(siehe 2.7.1). Also wird $\eta$ in die Eigenbündel $\eta_{+}$und $\eta_{-}$von $\Phi c_{\Omega}$ zerlegt, die zu den Eigenwerten +1 und -1 bzw. $+i$ und $-i$ gehören. In der Faser $\xi_{x}$ sei $v_{x}$ derart, daß $Q_{x}\left(v_{x}\right) \neq 0$ ist. Dann ist $\Phi_{x} v_{x}$ ein 
A. I. 521

Isomorphismus von $\eta_{x}$, der wegen $v_{x} c_{\Omega x}=-c_{\Omega x} v_{x}$ (siehe 2.7.1) die Eigenräume $\eta_{+x}$ und $\eta_{-x}$ vertauscht:

$$
\Phi_{x} v_{x}: \eta_{+x} \rightarrow \eta_{-x} \text { und } \eta_{-x} \rightarrow \eta_{+} \text {. }
$$

Insbesondere folgt $\operatorname{dim} \eta_{+}=\operatorname{dim} \eta_{-}$.

Beispiel 1. Sei $(\xi, Q)$ ein reelles $Q$-Bündel; dann hat

$$
(\xi, Q) \oplus(\xi,-Q)
$$

eine natürliche S-Struktur mit $\wedge \xi$ als Spinorbündel, in der die beiden Bündel der geraden und ungeraden Elemente von $\wedge \xi$ die Rolle der Halbspinorbündel spielen.

Um dies zu zeigen, seien $\left(\xi_{x}, Q_{x}\right), \wedge \xi_{x}$ die Fasern über $x \in M$ von $(\xi, Q)$ bzw. $\wedge \xi$. Unter Benützung der in 2.6 eingeführten Bezeichnungen ordnen wir dem Paar $\left(v_{x}, w_{x}\right) \in \xi_{x} \oplus \xi_{x}$ den Endomorphismus $\varphi_{x}\left(v_{x}, w_{x}\right)$ von $\wedge \xi_{x}$ zu:

$$
f_{x}\left(v_{x}, w_{x}\right)=L v_{x}+\partial v_{x}+L u_{x}-\partial w_{x} .
$$

$\varphi_{x}$ ist dann eine lineare Abbildung $\xi_{x} \oplus \xi_{x} \rightarrow$ End $\Lambda \xi_{x}$, von der man leicht nachweist, daß

$$
\left(p_{x}\left(v_{x}, w_{x}\right)\right)^{2}=\left(Q_{x}\left(v_{x}\right)-Q_{x}\left(w_{x}\right)\right) \mathrm{id}_{x}
$$

gilt. Nach $\mathrm{S} 1$ definiert somit das Paar $(\wedge \xi, \varphi)$ eine S-Struktur auf $(\xi, Q) \oplus(\xi,-Q)$. Da $\xi \oplus \xi$ eine natürliche Orientierung $\Omega$ hat, so ist $c_{\Omega}$ definiert. Man verifiziert nun noch, daß

$$
\Phi c_{\Omega}= \pm *^{2}
$$

wo * der dualisierende Operator in $\wedge \xi$ ist (vgl. 2.8). Somit ist die durch 3.2.1 gegebene Zerlegung diejenige in gerade und ungerade Elemente von $\wedge \xi$.

Beispiel 2. Die in 2.6 definierte Abbildung $\tilde{\mu}: \gamma \rightarrow$ End $\wedge \xi$ läßt das Cliffordbündel $\gamma$ von $(\xi, Q)$ auf dem äußeren Bündel operieren, definiert also eine $S$-Struktur $(\wedge \xi)_{\tilde{\mu}}$ auf $(\xi, Q)$. Ist $Q$ positiv-definit und $\operatorname{dim} \xi$ gerade $(=2 \mathrm{~m})$, dann ist in der Komplexifizierung $\wedge \xi_{C}$ von $\wedge \xi$ :

$$
\tilde{\mu} c_{\Omega}=i^{m} \alpha \quad(i=\sqrt{-1}),
$$

wo $\alpha$ die in [1], 1, S.426, definierte Abbildung ist.

3.3. Definition von SC-Strukturen. Ist $\eta$ ein Algebrabüridel, dann heißt ein $\eta$-Bündel $\alpha$ irreduzibel, falls jede Faser $\alpha_{x}$ ein im algebraischen Sinn irreduzibler $\eta_{x}$-Modul ist. Unter einer $S C$-Struktur auf $(\xi, Q)$ verstehen wir eine komplexe, irreduzible S-Struktur auf dem reellen $Q$-Bündel $(\xi, Q)$. Es gilt: 
3.3.1. $\sigma_{\Phi}$ sei eine SC-Struktur auf $(\xi, Q)$ und $m$ die ganze Zahl, mit der $\operatorname{dim} \xi=2 m$ oder $=2 m+1$ ist. Dann ist $\operatorname{dim} \sigma=2^{m}$ (über $\left.C\right)$.

Denn $\sigma_{\Phi}$ ist ein $\gamma$-Bündel, wo $\gamma$ das Cliffordbündel von $(\xi, Q)$ ist. Da $\sigma$ ein komplexes Vektorbündel ist, kann man $\sigma_{\Phi}$ auch als $\gamma \otimes C$ -Bündel auffassen $(\gamma \otimes C=$ Komplexifizierung von $\gamma)$. Nun ist jede Faser $(\gamma \otimes C)_{x}$ als Cliffordalgebra über den komplexen Zahlen entweder einfach ( $\operatorname{dim} \xi=2 m$ ) oder direkte Summe zweier gleichdimensionaler einfacher Ideale ( $\operatorname{dim} \xi=2 m+1$ ) (siehe [3], II.2.1, S. 42 und II.2.6, S. 47). Weiter ist $\operatorname{dim} \gamma=2^{n}$, mit $n=\operatorname{dim} \xi$.

Aus $\mathrm{S} 1$ in 3.2 folgt, daß jede SC-Struktur auf $(\xi, Q)$ durch folgende Daten gegeben werden kann:

SC 1. Ein komplexes Vektorbündel $\sigma$ der Dimension $2^{m}$, unter der Toraussetzung $\operatorname{dim} \xi=2 m$ oder $=2 m+1$;

SC 2. Eine Bündelabbildung $\varphi: \xi \rightarrow$ End $\sigma$ mit der Eigenschaft

$$
\left(\varphi_{x} v_{x}\right)^{2}=Q_{x}\left(v_{x}\right) \mathrm{id}_{x}, \quad v_{x} \in \xi_{x} .
$$

Aufgrund des Isomorphiesatzes für Cliffordbündel 2.4.3 schließt man:

3.3.2. $Q$ und $Q^{\prime}$ seien quadratische Formen auf dem reellen Vektorbündel $\xi$. Dann induziert jede SC-Struktur auf $(\xi, Q)$ eine solche auf $\left(\xi, Q^{\prime}\right)$ mit demselben Spinorbündel.

Dieser Satz ist insofern bedeutungsvoll, als er zəigt, daß die Existenz von SC-Strukturen auf dem reellen Vektorbündel $(\xi, Q)$ nur vom Vektorbündel $\xi$, aber nicht von der quadratischen Form $Q$ abhängt. Wir verstshen nun unter einem Spinorbündel des reellen Vektorbündels $\xi$ ein Spinorbündel einer SC-Struktur auf $(\xi, Q), Q$ bəliebig.

3.4. Existenz einer SC-Struktur. Mit der vorhin eingeführten Terminologie ist die Aussage, $(\xi, Q)$ habe für jede quadratische Form $Q$ eine SC-Struktur, gleichbedeutend mit der folgenden: das Vektorbündel $\xi$ hat ein Spinorbündel. Der Inhalt dieses Abschnittes besteht nun aus dem Beweis des folgenden Satzes:

3.4.1. Ein reelles und orientierbares Vektorbündel $\xi$ hat genau dann ein Spinorbündel, wenn die ganzzahlige Kohomologieklasse $W_{3}(\xi)=0$ ist.

Als Vorbereitung zum Beweis von 3.4.1 und unter den gleichen Annahmen zeigen wir vorerst:

3.4.2. $M$ sei die Basismannigfaltigkeit von $\xi$, und $\xi_{1}$ sei ein triviales reelles Geradenbündel über $M$. $\xi$ hat genau dann ein Spinorbündel, wenn dies für $\xi \oplus \xi_{1}$ zutrifft.

Beweis von 3.4.2. Der Beweis hat in beiden Richtungen ausgeführt zu werden, und außerdem hat man noch die Fallunterscheidung $\operatorname{dim} \xi$ gerade und $\operatorname{dim} \xi$ ungerade zu machen. Wir geben den Beweis nur für 
die Annahme: $\operatorname{dim} \xi$ sei ungerade und $\sigma$ ein Spinorbündel von $\xi \oplus \xi_{1}$. In den anderen Fällen verläuft er analog.

$Q$ und $Q_{1}$ seien quadratische Formen auf $\xi$ bzw. $\xi_{1}$, wobei $Q_{1}$ als negativ-definit vorausgesetzt wird. Das Cliffordbündel $\gamma$ von $(\xi, Q) \oplus\left(\xi_{1}, Q_{1}\right)$ operiert irreduzibel auf $\sigma$, die dem Element $c_{x} \in \gamma_{x}$ entsprechende Operation auf $\xi_{x} \oplus \xi_{1 x}\left(\gamma_{x}, \sigma_{x}=\right.$ Fasern in $\gamma$ bzw. $\sigma$ über $x \in M$ ) sei wieder mit $\Phi_{x} c_{x}$ bezeichnet. Nach 3.3.2 und 3.2.1 zerfällt $\sigma$ in die Eigenbündel $\sigma_{+}$und $\sigma$ - bezüglich der Operation $\Phi c_{\Omega}$. $\xi_{1}$ hat einen Schnitt $v_{1}$ mit der Eigenschaft $Q_{1 x}\left(v_{1 x}\right)=-1 . \Phi v_{1}$ ist dann ein Isomorphismus $\sigma \rightarrow \sigma$, der nach (1) in $3.2 \sigma_{+}$und $\sigma_{-}$ vertauscht. Ebenfalls aus (1) in 3.2 folgt, daß die dem Produkt $v_{x} v_{1 x}$ $\epsilon \gamma_{x}$, für $v_{x} \in \xi_{x}$, entsprechende Operation $\Phi_{x}\left(v_{x} v_{1 x}\right)$ sowohl $\sigma_{+x}$ als auch $\sigma_{-x}$ invariant läßt. Wir setzen nun

$$
\varphi_{x}^{\prime} v_{x}:=\Phi_{x}\left(v_{x} v_{1 x}\right)
$$

Dann gilt

$$
\left(\varphi_{x}^{\prime} v_{x}\right)^{2}=\Phi_{x}\left(v_{x} v_{1 x} v_{x} v_{1 x}\right)=\Phi_{x}\left(-v_{1 x}^{2} r_{x}^{2}\right)=Q_{x}\left(v_{x}\right) \text { id } \mathrm{r}_{-x}
$$

Ist $\operatorname{dim} \xi=2 m+1$, dann ist $\operatorname{dim} \sigma_{+}=2^{m}$. Also defjniert f $_{x}^{\prime}$ gemäß $\mathrm{SC} 1$ und SC 2 (vgl. 3.3) eine SC-Struktur auf $(\xi, Q)$.

Beweis von 3.4.1. Voraussetzung: 3.4.1 sei richtig für Vektorbündel einer gewissen Dimension $n \geqq 1$. Ist $\xi$ ein Vektorbündel der Dimension $n-1$ und $\xi_{1}$ ein triviales reelles Geradenbündel, dann ist $\operatorname{dim}\left(\xi \oplus \xi_{1}\right)$ $=n$ und $W_{3}\left(\xi \oplus \xi_{1}\right)=W_{3}(\xi)$. Aus 3.4 .2 folgt dann, daß 3.4 .1 richtig ist für Vektorbündel der Dimension $n-1$.

Es genügt also, 3.4.1 unter der Voraussetzung $\operatorname{dim} \xi=2 \mathrm{~m}$ mit $m \geqq 2$ zu beweisen. Es sei $V$ die typische Faser von $\xi, Q_{0}$ eine positivdefinite quadratische Form auf $V$, $S O$ die spezielle orthogonale Gruppe von $Q_{0}, C$ die komplexifizierte Cliffordalgebra ron $\left(V, Q_{0}\right)$ mit Automorphismengruppe Aut $C$.

Die Isomorphieklassen reeller Vektorbündel der Dimension $2 \mathrm{~m}$ über der festen Basismannigfaltigkeit $M$ sind die Elemente ron $H^{1}(S O)$, der ersten Kohomologiemenge von $M$ mit der Garbe der Keime lokaler differenzierbarer Funktionen auf $M$ mit Werten in $S O$ als Koeffizienten ([9], 3.2.a, S. 44; in der Bezeichnung von $H^{1}(S O)$ unterdrücken wir die Mannigfaltigkeit $M$, da sie im folgenden fest bleibt). Ebenso sind die Isomorphieklassen von komplexen Algebrabündeln über $M$ mit typischer Faser $C$ die Elemente von $H^{1}$ (Aut $C$ ).

Jedes $g \in S O$ wird zu einem Automorphismus $\varepsilon g \in$ Aut $C$ erweitert (Universalität der Cliffordalgebra, [3] II.1.1, S. 39). Die so definierte Injektion $\varepsilon: S O \rightarrow$ Aut $C$ induziert eine Abbildung 


$$
\varepsilon_{*}: H^{1}(S O) \rightarrow H^{1}(\text { Aut } C) .
$$

Ist $(\xi, Q)$ ein reelles $Q$-Bündel der Dimension $2 m$, dann ist die Isomorphieklasse $(\gamma)$ des komplexifizierten Cliffordbündels $\gamma$ von $(\xi, Q)$ das $\varepsilon_{*}$-Bild der Isomorphieklasse $(\xi)$ von $(\xi, Q)$ (vgl. 2.5).

Da $\operatorname{dim} V=2 m$ ist, so ist $C$ eine einfache komplexe Algebra, somit isomorph der Endomorphismenalgebra eines komplexen Vektorraumes $S$. Wir wollen hinfort $C$ mit End $S$ identifizieren; die invertiblen Elemente von $C$ bilden dann die volle lineare Gruppe $\mathrm{Gl}(S)$. Jedes $h \in \mathrm{Gl}(S)$ bestimmt einen inneren Automorphismus $\alpha h \in$ Aut $C$, und nach dem Satz von Nöther-Skolem ist $\alpha$ surjektiv. Man erhält somit eine exakte Sequenz von Gruppen

$$
0 \rightarrow C^{*} \rightarrow \mathrm{Gl}(S) \rightarrow \text { Aut } C \rightarrow 0
$$

$C^{*}$ ist die multiplikative Gruppe der komplexen Zahlen $\neq 0$.

Ein komplexes Vektorbündel $\sigma$ ist nun ein Spinorbündel von $(\xi, Q)$ (mit typischer Faser $S$ ), falls für die Isomorphieklassen gilt:

$$
(\gamma)=\varepsilon_{*}(\xi)=\alpha_{*}(\sigma) .
$$

Denn $\alpha_{*}(\sigma)$ ist die Isomorphieklasse des Endomorphismenbündels End $\sigma$, und $» \sigma$ ist Spinorbündel von $(\xi, \sigma)$ « bedeutet, daß End $\sigma$ isomorph $\gamma$ ist.

Wir haben noch weitere exakte Sequenzen zu betrachten. Es sei Spin die universelle Überlagerungsgruppe von $S O$. Da $\operatorname{dim} V \geqq 3$ angenommen wurde, so hat man die exakte Sequenz

$$
0 \rightarrow Z_{2} \rightarrow \operatorname{Spin} \rightarrow \mathrm{SO} \rightarrow 0
$$

Schließlich noch die exakten Sequenzen abelscher Gruppen:

$$
\begin{aligned}
& 0 \rightarrow Z \rightarrow C \stackrel{\beta}{\rightarrow} C^{*} \rightarrow 0, \\
& 0 \rightarrow Z \stackrel{\tau}{\rightarrow} \boldsymbol{Z} \rightarrow Z_{2} \rightarrow 0 .
\end{aligned}
$$

$\beta$ ist die Abbildung $z \rightarrow \varrho^{2 \pi i z}, \tau$ die Multiplikation mit 2 in $\boldsymbol{Z}$.

Die exakten Sequenzen (1) und (2) werden in ein kommutatives Diagramm eingebettet

$$
\begin{aligned}
& 0 \rightarrow C^{*} \rightarrow \operatorname{Gl}(S) \rightarrow \text { Aut } C \rightarrow 0 \\
& l_{1} \uparrow \quad l_{2} \uparrow \quad \varepsilon \uparrow \quad . \\
& 0 \rightarrow Z_{2} \rightarrow \text { Spin } \rightarrow \text { SO } \rightarrow 0
\end{aligned}
$$

$l_{1}$ und $l_{2}$ sind die natürlichen Inklusionen (Spin ist eine Untergruppe 2 
der invertiblen Elemente von $C$, Spin $=I_{0}^{+}$in der Terminologie von [4], III.6, S. 47). Ebenso die Sequenzen (3) und (4)

$$
\begin{aligned}
& 0 \rightarrow Z \rightarrow C \rightarrow C^{*} \rightarrow 0 \\
& \operatorname{id} \uparrow \quad \varrho \uparrow \quad l_{1} \uparrow \quad, \\
& 0 \rightarrow \boldsymbol{Z} \rightarrow \boldsymbol{Z} \rightarrow \boldsymbol{Z}_{2} \rightarrow 0
\end{aligned}
$$

wo $\varrho$ die Multiplikation mit $1 / 2$ ist.

Nun induziert bekanntlich [6] jede exakte Sequenz von Liegruppen $0 \rightarrow A_{1} \rightarrow A_{2} \rightarrow A_{3} \rightarrow 0$ eine exakte Sequenz der Kohomologiegruppen und -mengen

$$
H^{1}\left(A_{1}\right) \rightarrow H^{1}\left(A_{2}\right) \rightarrow H^{1}\left(A_{3}\right) \stackrel{\delta}{\rightarrow} H^{2}\left(A_{1}\right),
$$

falls $A_{1}$ abelsch ist. Diese Sequenz bricht nicht ab, falls auch $A_{2}$ und $A_{3}$ abelsch sind.

Die verbindenden Homomorphismen $\delta$ werden für die Sequenzen (1) ... (4) mit $\delta_{1} \ldots \delta_{4}$ bezeichnet; für den von einem Homomorphismus $v: A \rightarrow B$ induzierten Homomorphismus $H^{i}(A) \rightarrow H^{i}(B)$ wird $v_{*}$ gesetzt.

Den kommutativen Diagrammen (3.4.4) und (3.4.5) entsprechen also kommutative Diagramme der Kohomologiemengen und -gruppen mit den exakten Zeilen (3.4.6). Man hat insbesondere folgende Kommutationsregeln:

$$
\begin{aligned}
& \delta_{1} \varepsilon_{*}=l_{1 *} \delta_{2}, \\
& \delta_{3} l_{1 *}=\operatorname{id}_{*} \delta_{4} .
\end{aligned}
$$

Wir betrachten nun die Aussage

$$
\text { 3.4.9) } \xi \text { hat ein Spinorbündel. }
$$

Aus der Exaktheit von (3.4.6) und aus (3.4.3) folgt

$$
(3.4 .9) \Leftrightarrow \delta_{1}(\gamma)=0 .
$$

Mit (3.4.7) ergibt dies

$$
\text { (3.4.9) } \Leftrightarrow l_{1 *} \delta_{2}(\xi)=0 .
$$

Nun ist $\delta_{2}(\xi) \in H^{2}\left(\boldsymbol{Z}_{2}\right)$ die zweite Stiefel-Whitney-Klasse $w_{2}(\xi)$ [8]. Aus (3.4.8) folgt deshalb weiter

$$
\delta_{3} l_{1 *} w_{2}(\xi)=\delta_{4} w_{2}(\xi) .
$$

Nun ist $\delta_{3}$ ein Isomorphismus, also gilt

$$
(3.4 .9) \Leftrightarrow \delta_{4} w_{2}(\xi)=0,
$$

und nach Definition ist $W_{3}(\xi)=\delta_{4} w_{2}(\xi)$. 
3.5. Morita-Theorie und Eindeutigkeit des Spinorbündels. Es sei $\eta$ eine komplexe S-Struktur auf dem reellen $Q$-Bündel $(\xi, Q)$ mit Basismannigfaltigkeit $M, \Phi: \gamma \rightarrow$ End $\eta$ die Operation des komplexen Cliffordbündels $\gamma$ von $(\xi, Q)$ auf $\eta$. Ist $\varkappa$ ein komplexes Vektorbündel über $M$, so wird $\eta \otimes \varkappa$ mittels der Operation

$$
\gamma \rightarrow \text { End } \eta \rightarrow \text { End } \eta \otimes \text { End } x \rightarrow \text { End }(\eta \otimes x)
$$

zu einer S-Struktur auf $(\xi, Q)$. Somit ist $\eta \otimes$ ein Funktor von $V_{C}(M)$, der Kategorie der komplexen differenzierbaren Vektorbündel über $M$, in die Kategorie $\mathcal{V}_{\gamma}(\boldsymbol{M})$ der differenzierbaren $\gamma$-Bündel, d.h. der SStrukturen auf $(\xi, Q)$. Die Morita-Theorie sagt nun:

3.5.1. $\eta \otimes: \mathcal{V}_{C}(M) \rightarrow \mathcal{V}_{\gamma}(M)$ ist genau dann eine Äquivalenz, wenn $\eta$ eine SC-Struktur auf $(\xi, Q)$ ist.

Ist nämlich $F$ der Ring der differenzierbaren komplexwertigen Funktionen auf $M, A$ die $F$-Algebra der differenzierbaren Schnitte von $\gamma$, so ist $\eta$ genau dann eine SC-Struktur auf $(\xi, Q)$, wenn $A \cong$ End $\Gamma \eta$ (3.4.3); dies ist aber (da $\Gamma \eta$ endlich erzeugt, projektiv und ein Generator in $F-M o d$ ist) die Bedingung, daß $\Gamma \eta \otimes: F-M o d \rightarrow A-M o d$ eine Äquivalenz ist ([2], (4.4) Prop., S. 68).

Insbesondere folgt: $\mathrm{Zu}$ zwei SC-Strukturen auf $(\xi, Q), \sigma$ und $\sigma^{\prime}$, gibt es ein bis auf Isomorphie eindeutig bestimmtes komplexes Geradenbündel $\lambda$ mit $\sigma^{\prime}=\lambda \otimes \sigma$; ist $W_{3}(\xi)=0$, dann ist die Menge der SCStrukturen auf $(\xi, Q)$ gleichmächtig wie $H^{2}(M, Z)$.

3.6. SC-Strukturen auf komplexen Bündeln. Die Bedingung $W_{3}(\xi)=0$ für die Existenz eines Spinorbündels von $\xi$ impliziert beispielsweise, daß das reelle Tangentialbündel einer komplexer Mannigfaltigkeit SC-Strukturen zuläßt. In diesem Fall gibt es sogar ein ausgezeichnetes Spinorbündel, wie wir im folgenden unter etwas allgemeineren Annahmen zeigen werden.

Es sei $\xi_{C}$ ein komplexes Vektorbündel über der Basismannigfaltigkeit $M, \xi$ das reelle Bündel von $\xi_{C}$. Es gilt dann:

3.6.1. Das äußere Bündel $\wedge \xi_{C}$ ist Spinorbündel von $\xi$.

Um dies zu zeigen, sei $H$ eine nichtentartete Hermitesche Form auf $\xi_{C}, B$ der Realteil von $H, Q$ die zu $B$ assoziierte quadratische Form. $B$ und $Q$ sind Formen auf $\xi$.

Für $v_{x} \in \xi_{x}$ ist $w_{x} \mapsto H_{x}\left(v_{x}, w_{x}\right)$ eine lineare Funktion auf $\xi_{C x}$, die sich zu einer Antiderivation $\partial_{v_{x}}$ von $\wedge \xi_{C x}$ erweitert (vgl. 2.6). Die Zuordnung $\quad v_{x} \rightarrow \partial_{v_{x}}$ ist $\boldsymbol{R}$-linear, also eine lineare Abbildung $\xi_{x} \rightarrow$ End $\left(\wedge \xi_{C x}\right)$. Ist $L_{v_{x}}$ die Linksmultiplikation mit $v_{x}$ in $\wedge \xi_{C x}$, dann ist

$$
v_{x} \mapsto L_{v_{x}}+\partial_{v_{x}}
$$


eine lineare Abbildung $\varphi_{x}: \xi_{x} \rightarrow$ End $\left(\wedge \xi_{C x}\right)$. Man berechnet leicht, daß

$$
\left(\varphi_{x} v_{x}\right)^{2}=Q_{x}\left(v_{x}\right) \text { id }
$$

ist. Also definiert $\left(\wedge \xi_{C}, \varphi\right)$ nach SC 1 und SC 2, vgl. 3.3, eine SC-Struktur auf $(\xi, Q)$.

\section{Diracoperator}

4.1. Definition Hermitescher Formen auf S-Strukturen. Es sei $\eta_{\Phi}$ eine S-Struktur auf dem reellen $Q$-Bündel $(\xi, Q)$. Unter einer Hermiteschen Form (bzw. symmetrischen Bilinearform) $H$ auf $\eta_{\Phi}$ verstehen wir eine solche auf dem Vektorbündel $\eta$ mit der folgenden Eigenschaft:

A. Ist $\operatorname{dim} \xi$ gerade, dann ist für jeden Schnitt $v \in \Gamma \xi$ die Operation $\Phi v$ auf $\eta$ in der Metrik $H$ antiselbstadjungiert, d. h. es gilt

$$
H\left((\Phi v) s_{1}, s_{2}\right)=-H\left(s_{1},(\Phi v) s_{2}\right), s_{1}, s_{2} \in \Gamma \eta .
$$

Diese Eigenschaft bewirkt u.a., daß der Diracoperator selbstadjungiert wird (vgl. 4.8). Für ungerade Dimensionen von $\xi$ käme dann unter Umständen nur die Form $H \equiv 0$ in Frage. Wir verlangen deshalb:

B. Ist $\operatorname{dim} \xi$ ungerade, dann ist für jedes $v \in \Gamma \xi$ die Operation $\Phi v$ auf $\eta$ in der Metrik $H$ entweder selbstadjungiert oder antiselbstadjungiert.

Zusätzlich sei $\nabla$ ein linearer Zusammenhang auf $(\xi, Q)$ (siehe 2.9.1). Dann versteh€n wir unter einem linearen Zusammenhang $\nabla^{\prime}$ auf $\left(\nabla, \eta_{\Phi}\right)$ einen solchen auf dem Vektorbündel $\eta$ mit der Eigenschaft:

4.1.1. $\nabla_{h}^{\prime} \cdot((\Phi v) s)=\left(\Phi\left(\nabla_{h} \cdot v\right) s+(\Phi v)\left(\nabla_{h}^{\prime} \cdot s\right)\right.$ für alle $h \in \mathcal{X}$, $v \in \Gamma \xi, s \in \Gamma \eta$.

Dies bedeutet, daß der von $\nabla$ und $\nabla^{\prime}$ auf $\operatorname{Hom}(\gamma$, End $\eta)$ induzierte lineare Zusammenhang den Wert 0 auf $\Phi: \xi$ hat.

4.1.2. $\xi$ sei orientiert und das Spinorbündel $\eta$ der S-Struktur $\eta_{\Phi}$ auf $(\xi, Q)$ zerfalle in die Halbspinorbündel $\eta_{+}$und $\eta_{-}$. Jeder lineare Zusammenhang $\nabla^{\prime}$ auf $\left(\nabla, \eta_{\Phi}\right)$ läßt die Zerlegung $\eta_{-}=\eta_{+} \oplus \eta_{-}$invariant.

Beweis. $\eta_{+}$und $\eta_{-}$sind die Eigeribündel des Operators $\Phi c_{\Omega}$ (3.2.1) zu den Eigenwerten \pm 1 bzw. $\pm i$. Aus 4.1.1 und der lokalen Darstellbarkeit durch ein Produkt:

$$
c_{\Omega}=v_{1} \ldots v_{n} \quad\left(v_{k} \text { lokale Schnitte in } \xi\right. \text {, vgl. 2.7, (1)) }
$$

folgt die Beziehung

$$
\nabla_{h}^{\prime} \cdot\left(\left(\varphi c_{\Omega}\right) s\right)=\varphi\left(\nabla_{h} \cdot c_{\Omega}\right) s+\left(\varphi c_{\Omega}\right)\left(\nabla_{h}^{\prime} \cdot s\right)
$$

also wegen $\nabla \cdot c_{\Omega}=0 \quad(2.9 .2)$ : 


$$
\nabla_{h}^{\prime} \cdot\left(\left(\varphi c_{\Omega}\right) s\right)=\left(\varphi c_{\Omega}\right)\left(\nabla_{h}^{\prime} \cdot s\right)
$$

Somit ist $\nabla_{h}^{\prime} \cdot s$ wieder ein »Eigenvektor« von $\varphi c_{\Omega}$, falls $s$ ein solcher ist, und zwar zum gleichen Eigenwert wie $s$.

Für eine SC-Struktur $\sigma_{\Phi}$ auf $(\xi, Q)$ (vgl. 3.3) gelten folgende Eindeutigkeitssätze:

E 1. Sei $\left\{\sigma_{\Phi}\right\}_{H}$ die Gesamtheit aller Hermiteschen Formen auf $\sigma_{\Phi}$. Ist $H \in\left\{\sigma_{\Phi}\right\}_{H}$, dann auch $\lambda H \in\left\{\sigma_{\Phi}\right\}_{H}$ für jede reelle Funktion $\lambda \in \mathcal{F}_{R}$; ist $H_{1}$ nichtentartet, dann ist $\left\{\sigma_{\Phi}\right\}_{H}=\mathcal{F}_{R} H_{1}$.

E 2. Sei $\left\{\nabla, \sigma_{\Phi}\right\}_{\nabla}$ die Gesamtheit aller linearer Zusammenhänge auf $\left(\nabla, \sigma_{\Phi}\right)$, und $\nabla^{\prime} \in\left\{\nabla, \sigma_{\Phi}\right\}_{\nabla}$. Dann ist $\left\{\nabla, \sigma_{\Phi}\right\}_{\nabla}=\nabla^{\prime}+\mathcal{X}_{C}^{*}$, $x_{C}^{*}=$ linearer Raum der komplexwertigen 1 -Formen auf $M$.

Als Illustration beweisen wir E 1. Der erste Teil der Behauptung ist trivial. Zum zweiten Teil: $\mathrm{Da} H_{1}$ njchtentartet ist, so existiert zu jedem $H \in\left\{\sigma_{\Phi}\right\}_{H}$ eine in $H_{1}$ selbstadjungierte Abbildung $\psi: \sigma \rightarrow \sigma$ derart, daß

$$
H_{x}\left(s_{1 x}, s_{2 x}\right)=H_{1 x}\left(\psi_{x} s_{1 x}, s_{2 x}\right)
$$

für alle $s_{1 x}, s_{2 x} \in \sigma_{x}$ (= Faser von $\sigma$ über $x \in M$ ) ist. Die Bedingung, $\varphi_{x} v_{x}$ für $v_{x} \in \xi_{x}$ sei sowohl in $H_{x}$ als auch in $H_{1 x}$ (anti-)selbstadjungiert, drückt sich durch die Gleichung

$$
\left(\varphi_{x} v_{x}\right) \psi_{x}=\psi_{x}\left(\varphi_{x} v_{x}\right)
$$

aus. Diese Gleichung gilt aber auch, wenn $v_{x}$ durch ein $c_{x}$ in der Faser $\gamma_{x}$ des Cliffordbündels von $(\xi, Q)$ ersetzt wird, denn $\xi_{x}$ erzeugt $\gamma_{x}$. Da nun $\varphi_{x}: \gamma_{x} \rightarrow$ End $\sigma_{x}$ komplex irreduzibel ist, folgt $\psi_{x}=\lambda_{x} \mathrm{id}_{n_{x}}$ mit $\lambda_{x} \in R$.

Ebenso wird im Beweis von $\mathrm{E} 2$ die Irreduzibilität von $\varphi_{x}$ benützt.

4.2. Existenz Hermitescher Formen auf SC-Strukturen. Die bisherigen Ausführungen in den Kapiteln 3 und 4 betrafen orientierte reelle Vektorbündel, während in Kapitel 2 die Orientierung keine wesentliche Rolle spielte. Beispielsweise ist Satz 2.2.2 über die definite Zerlegung reeller $Q$-Bündel nicht richtig im Rahmen der orientierten Vektorbündel. Wir betrachten nun im folgenden nur orientierte reelle $Q$-Bündel $(\xi, Q)$ (alle über derselben Basismannigfaltigkeit $M$ ), deren Summanden in der definiten Zerlegung

$$
(\xi, Q)=\left(\xi_{+}, Q_{+}\right) \oplus\left(\xi_{-}, Q_{-}\right)
$$

wieder orientiert sind.

Unter diesen Voraussetzungen gilt nun:

4.2.1. $\sigma_{\Phi}$ sei eine SC-Struktur auf $(\xi, Q)$. Es gibt immer nichtentartete 
Hermitesche Formen auf $\sigma_{\Phi}$. Ist $t$ der Index von $Q$, dann sind diese Formen entweder definit (für $t=\operatorname{dim} \xi$, oder $t=0$ und $\operatorname{dim} \xi$ ungerade) oder vom Index $(\operatorname{dim} \sigma) / 2$. Hat $\xi$ gerade Dimension, dann sind bezüglich dieser Formen die Halbspinorbündel $\sigma_{+}$und $\sigma_{-}$entweder orthogonale Komplemente (dies für geraden Index $t$ ) oder total isotrop.

Beweis. $\left(\xi_{+}, Q_{+}\right) \oplus\left(\xi_{-}, Q_{-}\right)$sei die definite Zerlegung von $(\xi, Q)$, $U \subset M$ eine offene Menge derart, daß die Restriktionen $\xi_{+} \mid U$ und $\xi_{-} \mid U$ trivial sind. Es gibt dann Schnitte $v_{1}, \ldots, v_{n}(n=\operatorname{dim} \xi)$ in $\xi \mid U$ mit den Eigenschaften

$$
B\left(v_{v}, v_{\mu}\right)= \pm \delta_{v_{\ell \ell}}
$$

( $B$ die zu $Q$ assoziierte Bilinearform), wobei $v_{1}, \ldots, v_{r}, r=n-t$, eine Basis von $\xi_{+} \mid U, v_{r+1}, \ldots, v_{n}$ eine solche von $\xi_{-} \mid U$ bilden. Ist $\gamma$ das Cliffordbündel von $(\xi, Q)$, dann genügen die $v_{\nu}$, als Schnitte von $\gamma \mid U$ aufgefaßt, den Antikommutationsregeln

$$
v_{\nu} v_{\mu}+v_{\mu} v_{\nu}= \pm 2 \delta_{\nu \mu} 1 \quad(1=\text { Eins-Schnitt in } \gamma \mid U),
$$

die man aus (1) in 2.4 durch Polarisation erhält.

Daraus folgt, daß alle aus den Faktoren $v_{v}$ gebildeten Produkte in $\Gamma(\gamma \mid U)$ eine endliche Gruppe $G$ von Schnitten ausmachen. $G$ operiert vermittels $\Phi$ auf $\sigma \mid U$; ist $H_{U}$ eine beliebige positiv-definite Hermitesche Form auf $\sigma \mid U$, dann ist

$$
H_{U}^{\prime}\left(s_{1}, s_{2}\right):=\sum_{g \in G} H_{U}\left((\Phi g) s_{1},(\Phi g) s_{2}\right)
$$

eine bezüglich der Gruppe $\Phi G$ invariante, positiv-definite Hermitesche Form auf $\sigma \mid U$. Aus $\left(v_{v}\right)^{2}=1$ für $v_{v} \in \Gamma \xi_{+} \mid U$ folgt nun, daß $\Phi v_{v}$ (und somit $\Phi v$ für jedes $v \in \Gamma \xi_{+} \mid U$ ) selbstadjungiert in $H_{U}^{\prime}$ ist. Aus $\left(v_{\mu}\right)^{2}=-1$ für $v_{\mu} \in \Gamma \xi_{-} \mid U$ folgt ebenso, daß $\Phi v$ für jedes $v \in \Gamma \xi_{-} \mid U$ antiselbstadjungiert in $H_{U}^{\prime}$ ist.

Diese lokalen Formen lassen sich nun unter Benützung einer Partition der Eins zu einer globalen positiv-definiten Hermiteschen Form $H^{\prime}$ auf $\sigma$ zusammenfügen derart, daß $\Phi v$ für $v \in \Gamma \xi_{+}$selbstadjungiert, für $v \in \Gamma \xi^{-}$aber antiselbstadjungiert in $H^{\prime}$ ist.

Im folgenden werden wir nun drei Fälle unterscheiden:

F 1. $r=n-t$ sei gerade $\left(n=\operatorname{dim} \xi, t=\operatorname{Index} Q=\operatorname{dim} \xi_{-}\right)$. Das Cliffordbündel $\gamma_{+}$von $\left(\xi_{+}, Q_{+}\right)$wird als kanonisch in $\gamma$ eingebettet angenommen. Sei $\Omega_{+}$die Orientierung von $\xi_{+}, c_{+}=c_{\Omega+}$ der entsprechende kanonische Schnitt, nun als solcher von $\gamma$ aufgefaßt (vgl. 2.7). Aus der lokalen Darstellbarkeit von $c_{+}$:

$$
c_{+}=v_{1} \ldots v_{r}
$$


durch eine in $\Omega_{+}$positiv orientierte, orthonormierte Basis $\left\{v_{v}\right\}$ von $\xi+$ folgt dann

$$
\begin{aligned}
& v_{+} c_{+}+c_{+} v_{+}=0, \\
& v_{-} c_{+}-c_{+} v_{-}=0,
\end{aligned}
$$

$v_{+} \in \Gamma \xi_{+}, v_{-} \in \Gamma \xi_{-}$, die Multiplikation wird in $\gamma$ vollzogen.

Sei $\tilde{\alpha}$ die Adjungierte einer Abbildung $\alpha: \sigma \rightarrow \sigma$ in der Metrik $H^{\prime}$, $q$ die ganze Zahl mit $r=2 q$, und es sei $d_{+}=i^{q} \Phi c_{+}(i=\sqrt{-1})$ gesetzt. Dann folgt aus (1)

$$
\tilde{d}_{+}=d_{+},\left(d_{+}\right)^{2}=\mathrm{id}_{\sigma} .
$$

Also liefert die definierende Gleichung

$$
H\left(s_{1}, s_{2}\right):=H^{\prime}\left(d_{+} s_{1}, s_{2}\right), \quad s_{1}, s_{2} \in \Gamma \sigma,
$$

eine nichtentartete Hermitesche Form $H$ auf $\sigma$, in der wegen (2) $\Phi v$ antiselbstadjungiert ist für alle $v \in \Gamma \xi$. Also ist $H$ eine Hermitesche Form auf $\sigma_{\Phi}$. Ist $r=n-t \neq 0$, dann folgt aus (2) und (3), daß $d_{+}$zwei Eigenbündel gleicher Dimension zu den Eigenwerten \pm 1 bat, woraus dann Index $H=(\operatorname{dim} \sigma) / 2$ folgt. Für $r=0$ ist natürlich $H=H^{\prime}$ positiv-definit.

F 2. $r=n-t$ und $t$ seien ungerade. Dieselben Überlegungen wie vorhin, statt auf $\left(\xi_{+}, Q_{+}\right)$nun aber auf $\left(\xi_{-}, Q_{-}\right)$angewandt, liefern wiederum eine nichtentartete Hermitesche Form $H$ auf $\sigma$, in der alle $\Phi v$ antiselbstadjungiert sind.

F 3. $r$ sei ungerade und $t$ gerade. Wie vorhin erhält man eine nichtentartete Hermitesche Form auf $\sigma$, in der alle $\Phi v$ selbstadjungiert sind.

Damit ist der Existenzbeweis erbracht. Der zweite Teil der Behauptung 4.2.1 ergibt sich durch Vergleichen der beiden Schnitte $d_{+}$(bzw. $d_{-}$) und $c_{+}$, die einerseits verantwortlich sind für die Form $H^{\prime}$, andererseits für die Halbspinorbündel, unter Berücksichtigung der lokalen Produktdarstellungen (1) und (2) in 2.7.

4.3. Existenz linearer Zusammenhänge auf $\left(\nabla, \sigma_{\Phi}\right) .(\xi, Q)$ sei ein reelles $Q$-Bündel, $\nabla$ ein linearer Zusammenhang auf $(\xi, Q)$ (vgl. 2.9), und $\sigma_{\Phi}$ eine SC-Struktur auf $(\xi, Q)$. Wir zeigen:

4.3.1. Es gibt lineare Zusammenhänge auf $\left(\nabla, \sigma_{\Phi}\right)$.

Beweis. Es sei vorerst $\operatorname{dim} \xi$ gerade. Das komplexifizierte Cliffordbündel $\gamma_{C}$ von $(\xi, Q)$ hat dann Fasern, die einfache Algebren sind, und $\varphi_{C}: \gamma_{C} \rightarrow$ End $\sigma$ ist somit ein Isomorphismus der Algebrabündel.

Gemäß 2.9 kann man $\nabla$ zu einem linearen Zusammenhang auf 
$\gamma$ und dann zu einem solchen, $\widetilde{\nabla}$, auf $\gamma_{C}$ erweitern. Der Isomorphismus $\Phi_{C}: \gamma_{C} \rightarrow$ End $\sigma$ führt $\widetilde{\nabla}$ in einen linearen Zusammenhang $\varphi_{C} \widetilde{\nabla}$ auf End $\sigma$ über.

4.3.2. $\nabla^{\prime}$ sei ein linearer Zusammenhang auf $\sigma, \widetilde{\nabla}^{\prime}$ der von ihm auf End $\sigma$ induzierte. $\nabla^{\prime}$ ist genau dann ein linearer Zusammenhang auf $\left(\nabla, \sigma_{\Phi}\right)$ (Definiton 4.1.1), wenn $\widetilde{\nabla}^{\prime}=\Phi_{C} \widetilde{\nabla}$.

Dies zeigt eine einfache Rechnung. Der Beweis von 4.3.1 ist erbracht, falls folgendes gezeigt werden kann:

4.3.3. Jeder lineare Zusammenhang auf End $\sigma$ wird von einem solchen auf $\sigma$ induziert.

Den Beweis davon bringen wir in 4.4.

Für ungerade Dimensionen von $\xi$ geht man folgendermaßen vor. $\left(\xi_{1}, Q_{1}\right)$ sei ein eindimensionales triviales, reelles $Q$-Bündel mit linearem Zusammenhang. Man zeigt: Ist $\sigma$ das Spinorbündel der SC-Struktur $\sigma_{\Phi}$ von $(\xi, Q)$, sind $\sigma_{+}$und $\sigma_{-}$zwei Exemplare von $\sigma$, dann läßt sich eine SC-Struktur $\sigma_{\Phi^{\prime}}^{\prime}$ von $(\xi, Q) \oplus\left(\xi_{1}, Q_{1}\right)$ angeben mit Spinorbündel $\sigma^{\prime}=\sigma_{+} \oplus \sigma_{-}$, wobei $\sigma_{+}$und $\sigma_{-}$als deren Halbspinorbündel auftreten; ist ${ }_{2} \nabla$ der aus $\nabla$ auf $(\xi, Q)$ und ${ }_{1} \nabla$ auf $\left(\xi_{1}, Q_{1}\right)$ konstruierte lineare Zusammenhang auf $(\xi, Q) \oplus\left(\xi_{1}, Q_{1}\right), \quad \nabla^{\prime}$ derjenige auf $\left({ }_{2} \nabla, \sigma_{\Phi^{\prime}}^{\prime}\right)$, dessen Existenz bereits bewiesen worden ist, dann induziert $\nabla^{\prime}$ einen linearen Zusammenhang auf $\left(\nabla, \sigma_{\Phi}\right)$ mit Spinorbündel $\sigma=\sigma_{+}$.

4.4. Beweis von Hilssatz 4.3.3. $\sigma$ sei ein Vektorbündel über der Basismannigfaltigkeit $M$. Wir führen zwei neue Vektorbündel ein:

$\operatorname{End}_{0} \sigma:$ die Faser $\left(\operatorname{End}_{0} \sigma\right)_{x}, x \in M$, besteht aus allen Endomorphismen $t_{x}$ von $\sigma_{x}$ mit $\operatorname{Spur} t_{x}=0$.

Der $\sigma$ : die Faser $(\text { Der } \sigma)_{x}$ besteht aus allen Derivationen $d_{x}$ von $(\text { End } \sigma)_{x}$.

Es gibt eine natürliche Abbildung $\alpha: \operatorname{End}_{0} \sigma \rightarrow \operatorname{Der} \sigma$, die jedem $t_{0} \in \Gamma \operatorname{End}_{0} \sigma$ die innere Derivation

$$
\left(\alpha t_{0}\right) t=t_{0} t-t t_{0}, \quad t \in \Gamma \text { End } \sigma,
$$

zuordnet. Wegen der Normierung durch die Spur ist $\alpha$ ein Isomorphismus.

Nun sei $\nabla$ ein linearer Zusammenhang auf End $\sigma, \nabla^{\prime}$ ein solcher auf $\sigma$, und $\widetilde{\nabla}^{\prime}$ sei der durch $\nabla^{\prime}$ auf End $\sigma$ Induzierte. Die Differenz $\nabla-\widetilde{\nabla}^{\prime}$ ist bekanntlich eine Abbildung $\nabla-\widetilde{\nabla}^{\prime}: \tau(M) \rightarrow$ End End $\sigma$. Tatsächlich ist sie sogar wegen der Derivationseigenschaft c) in 4.1 eine Abbildung $\tau(M) \rightarrow$ Der $\sigma$. Die Komposition $\alpha^{-1}\left(\nabla-\widetilde{\nabla}^{\prime}\right)$ sei mit $\beta$, das Bild eines Vektorfeldes $h \in \mathcal{X}$ unter $\beta$ mit $\beta_{h}$ bezeichnet. Für jeden Schnitt $t \in \Gamma$ End $\sigma$ gilt dann wegen (1): 


$$
\nabla_{h} \cdot t-\widetilde{\nabla}_{h}^{\prime} \cdot t=\beta_{h} t-t \beta_{h}
$$

Auf $\sigma$ betrachten wir nun den neuen linearen Zusammenhang

$$
\nabla_{h}^{\prime \prime} \cdot s:=\nabla_{h}^{\prime} \cdot s+\beta_{h} s, s \in \Gamma \sigma .
$$

Für den durch ihn auf End $\sigma$ Induzierten gilt:

$$
\widetilde{\nabla}_{h}^{\prime \prime} \cdot t=\widetilde{\nabla}_{h}^{\prime} \cdot t+\beta_{h} t-t \beta_{h},
$$

somit wegen (2):

$$
\widetilde{\nabla}^{\prime \prime}=\nabla
$$

also induziert $\nabla^{\prime \prime}$ den gegebenen linearen Zusammenhang $\nabla$ auf End $\sigma$.

4.5. Vollständige S-Strukturen. Unter einer vollständigen S-Struktur $\left(\nabla, \eta_{\Phi}, \nabla^{\prime}, H\right)$ auf dem reellen $Q$-Bündel $(\xi, Q)$ verstehen wir eine S-Struktur $\eta_{\Phi}$ auf $(\xi, Q)$ (vgl. 3.2) zusammen mit folgenden Daten: einer nichtentarteten $\mathrm{H} \in \mathrm{rmiteschen}$ (falls $\eta$ komplex ist) bzw. einer symmetrischen Bilineareform (falls $\eta$ reell ist) auf $\eta_{\Phi}$ (vgl. 4.1), einem linearen Zusammenhang $\nabla$ auf $(\xi, Q)$ und einem solchen, $\nabla^{\prime}$, auf $\left(\nabla, \eta_{\Phi}\right)$ (vgl. 4.1) derart, daß $H$ und $\nabla^{\prime}$ die Ricci-Identität (2.9.1) erfüllen. Die letzte Forderung impliziert, daß $\nabla^{\prime}$ in $\eta_{\Phi}$ metrisierbar ist.

4.5.1. Sei $\nabla$ ein linearer Zusammenhang und $\sigma_{\Phi}$ eine SC-Struktur auf $(\xi, Q)$. Dann gibt es zu jeder nichtentarteten Hermiteschen Form $H$ auf $\sigma_{\Phi}$ einen linearen Zusammenhang $\nabla^{\prime}$ auf $\left(\nabla, \sigma_{\Phi}\right)$, soda $\beta$ diese GröBen eine vollständige SC-Struktur auf $(\xi, Q)$ bilden.

Beweis. Die linke Seite von Gleichung (2.9.1) ist definitionsgemäß die kovariante Ableitung der Form $H$ in Richtung des Vektorfeldes $h \in \mathcal{X}$. Es sei $H$ die gegebene nichtentartete Hermitesche Form auf $\sigma_{\Phi}$ und ${ }_{0} \nabla^{\prime}$ ein linearer Zusammenhang auf $\left(\nabla, \sigma_{\Phi}\right)$; dann zeigt man leicht, daß ${ }_{0} \nabla_{h}^{\prime} \cdot H$ wieder eine Hermitesche Form auf $\sigma_{\Phi}$ ist. Nach E 1 in 4.1 gibt es somit eine reellwertige 1-Form $\omega$ auf $M$, sodaß ${ }_{0} \nabla_{h}^{\prime} \cdot H=(\omega h) H$ st. Nach E 2 in 4.1 ist $\nabla^{\prime}$, gegeben durch

$$
\nabla_{h}^{\prime} \cdot s={ }_{0} \nabla_{h}^{\prime} \cdot s+\frac{1}{2}(\omega h) s, \quad s \in \Gamma \sigma
$$

wieder ein linearer Zusammenhang auf $\left(\nabla, \sigma_{\Phi}\right)$, der nun $\epsilon \mathbf{b}$ ən die Eigenschaft $\nabla^{\prime} \cdot H=0$ hat.

Allerdings ist umgekehrt nicht jeder lineare Zusammenhang auf $\left(\nabla, \sigma_{\Phi}\right)$ in $\sigma_{\Phi}$ metrisierbar. Es gilt nämlich:

4.5.2. Die Differenz ${ }_{1} \nabla^{\prime}-{ }_{2} \nabla^{\prime}$ zweier in $\sigma_{\Phi}$ metrisierbarer linearer Zusammenhänge auf $\left(\nabla, \sigma_{\Phi}\right)$ ist eine komplexwertige 1-Form auf $M$ (E 2 in 4.1), deren Realteil das Differential einer Funktion $\% \in \mathcal{I}_{\boldsymbol{R}}$ ist. 
Ist also $\nabla^{\prime}$ ein in $\sigma_{\Phi}$ metrisierbarer linearer Zusammenhang auf $\left(\nabla, \sigma_{\Phi}\right)$ und $\omega$ eine reelle 1 -Form auf $M$ mit $d \omega \neq 0$, dann ist $\nabla^{\prime}+\omega$ wieder ein linearer Zusammenhang auf $\left(\nabla, \sigma_{\Phi}\right)$ (E 2 in 4.1 ), der aber gemäß 4.5.2 nicht in $\sigma_{\Phi}$ metrisierbar ist.

4.6. S-Strukturen auf Riemannschen Mannigfaltigkeiten. Unter einer Riemannschen Mannigfa tigkeit $R$ verstehen wir ein $\operatorname{Paar} R=(M, Q)$, wo $M$ eine reelle differenzierbare Mannigfaltigkeit und $Q$ eine auf dem kontravarianten Tangentialbündel $\tau$ von $M$ definierte nichtentartete quadratische Form ist. Außerdem setzen wir wie bisher voraus, $M$ sei zusammenhängend. Ist $R=(M, Q)$, dann setzen wir: $-R:=(M,-Q)$. Durch $Q$ wird auf dem kovarianten Tangentialbündel $\tau^{*}$ von $M$ eine quadratische Form mit gleichem Index wie $Q$ induziert, für die wir ebenfalls das Symbol $Q$ verwenden. Das kontra-(ko-)variante Tangentialbündel von $R$ ist dann das reelle $Q$-Bündel $\tau(R):=(\tau, Q)$ (bzw. $\left.\tau^{*}(R):=\left(\tau^{*}, Q\right)\right)$.

Unter einer S-Struktur auf $R=(M, Q)$ verstehen wir nun eine solche auf dem kovarianten Tangentialbündel $\tau^{*}(-R)=\left(\tau^{*},-Q\right)$. Analog sind mit vollständigen S-Strukturen auf $R$, SC-Strukturen auf $R$, immer die entsprechenden Strukturen auf $\tau^{*}(-R)$ gemeint.

Die Wahl von $-Q$ rechfertigt sich aus verschiedenen Gründen; beispielsweise werden alle nichtentarteten Hermiteschen Formen einer SCStruktur auf einer positiv-definiten Riemannschen Mannigfaltigkeic wieder definit (4.2.1). Auf den beiden Tangentialbündeln einer Riemannschen Mannigfaltigkeit $R$ gibt es einen ausgezeichneten linearen Zusammenhang, nämlich denjenigen von Levi-Civiti, dessen Torsion Null ist. Unter einem linearen Zusammenhang auf einer S-Strulitur $\eta_{\Phi}$ auf $R$ rerstehen wir in Zukunft immer einen solchen auf $\left(\nabla, \eta_{\Phi}\right)$ mit $\nabla$ als Leri-CivitàZusammenhang. Entsprechend brauchen wir dann für eine rollständige S-Struktur auf einer Riemannschen Mannigfaligkeit $R$ nur noch drei Daten, $\eta_{\Phi}, \nabla^{\prime}, H$, anzugeben.

Wir bemerken noch, daß in einer S-Struktur $\eta_{\Phi}$ auf $R=(M, Q)$ die kovarianten Tangentialvektoren von $I I$ auf clem Spinorbündel $\eta$ operieren. Denn nach Definition (vgl. 3.2) läßt $\Phi$ das Cliffordbündel $\gamma$ von $\left(\tau^{*},-Q\right)$ auf $\eta$ operieren, also auch das Teilbündel $\tau^{*}$ von $\gamma$.

4.7. Definition des Diracoperators. Sei $\eta_{\Phi}$ eine S-Struktur auf der Riemannschen Mannigfaltigkeit $R=(M, Q)$ und $\nabla^{\prime}$ ein beliebiger linearer Zusammenhang auf dem Spinorbündel $\eta$. Jeder Schnitt $s \in \Gamma \eta$ bestimmt dann ein gemischtes Tensorfeld $t_{s}$ auf $M$ mit Werten in $\eta$ :

$$
t_{s}\left(h^{*}, h\right):=\left(\varphi h^{*}\right)\left(\nabla_{h}^{\prime} \cdot s\right), \quad h^{*} \in \mathcal{X}^{*} *, \quad h \in \mathcal{X} .
$$


Dessen Kontraktion über das Argumentenpaar $\left(h^{*}, h\right)$ liefert einen Schnitt $D s \in \Gamma \eta$, der lokal mittels einer Basis $\left\{h_{\nu}\right\}$ von $\tau$ und der dazu Dualen $\left\{h^{* \nu}\right\}$ von $\tau^{*}(v=1, \ldots, n=\operatorname{dim} M)$ in der Form

$$
D s=\sum_{\nu=1}^{n}\left(\Phi h^{* \nu}\right)\left(\nabla_{h}^{\prime} \cdot s\right)
$$

darstellbar ist.

Bei gegebener S-Struktur $\eta_{\Phi}$ auf $R$ definiert so jeder lineare Zusammenhang $\nabla^{\prime}$ auf dem Vektorbündel $\eta$ einen linearen Differentialoperator erster Ordnung $D: \Gamma \eta \rightarrow \Gamma \eta$. Ist $\left(\eta_{\Phi}, \nabla^{\prime}, H\right)$ eine vollständige S-Struktur auf $R$, dann ist der so konstruierte Operator $D$ der Diracoperator von $\left(\eta_{\Phi}, \nabla^{\prime}, H\right)$.

4.7.1. Hat $\left(\eta_{\Phi}, \nabla^{\prime}, H\right)$ zwei Halbspinorbündel $\eta_{+}$und $\eta_{-}$, dann induziert der Diracoperator $D$ von $\left(\eta_{\Phi}, \nabla^{\prime}, H\right)$ lineare Differentialoperatoren erster Ordnung

$$
\begin{aligned}
& D_{+}: \Gamma \eta_{+} \rightarrow \Gamma \eta_{-}, \\
& D_{-}: \Gamma \eta_{-} \rightarrow \Gamma \eta_{+} .
\end{aligned}
$$

Dies folgt aus der expliziten Darstellung (1) unter Berücksichtigung von (1) in 3.2 und 4.1.2.

4.8. Selbstadjungiertheit des Diracoperators. Es sei $R=(M, Q)$ eine Riemannsche Mannigfaltigkeit mit vollständiger S-Struktur $\left(\eta_{\Phi}, \nabla^{\prime}, H\right)$. Wir werden uns der Einfachheit halber auf gerade Dimensionen von $R$ beschränken, obschon die folgenden Ausführungen nach Änderung einiger Vorzeichen auch für ungerade Dimensionen von $R$ richtig wären.

Zwei Schnitte $s_{1}, s_{2} \in \Gamma \eta$ definieren durch die Gleichung

$$
H\left(\left(\Phi h^{*}\right) s_{1}, s_{2}\right)=\left\langle h^{*}, j\left(s_{1}, s_{2}\right)\right\rangle, \quad h^{*} \in \mathcal{C}^{*},
$$

ein komplexes kontravariantes Vektorfeld $j\left(s_{1}, s_{2}\right)$ auf $M$. Dabei bedeutet $\langle$,$\rangle das duale Produkt auf x_{C}^{*} \times x_{C}$, wo $x_{C}^{*}, x_{C}$ die Moduln der komplexwertigen ko- bzw. kontravarianten Vektorfelder auf $M$ sind. $j$ ist dann eine bilineare Bündelabbildung von $\eta \times \eta$ in das komplexifizierte kontravariante Tangentialbündel $\tau_{C}$ von $M$.

$j$ hat die Symmetrieeigenschaft

$$
\begin{aligned}
& j\left(s_{1}, s_{2}\right)=-j\left(s_{2}, s_{1}\right) \quad \text { falls } \eta \text { reell ist, } \\
& j\left(s_{1}, s_{2}\right)=-\overline{j\left(s_{2}, s_{1}\right)} \text { falls } \eta \text { komplex ist, }
\end{aligned}
$$

( $h \rightarrow \bar{h}$ Übergang zum Konjugiertkomplexen). Denn nach Definition in 4.1 ist $H$ eine Form auf $\eta_{\Phi}$, wenn 


$$
H\left(\left(\Phi h^{*}\right) s_{1}, s_{2}\right)=-H\left(s_{1},\left(\Phi h^{*}\right) s_{2}\right)
$$

ist.

Wir berechnen nun $\operatorname{div} j\left(s_{1}, s_{2}\right)$. Sei $\left\{h_{v}\right\}$ eine lokale Basis von $\tau$, $\left\{h^{* v}\right\}$ die dazu Duale und $\nabla$ der Levi-Cività-Zusammenhang auf $R$. Dann ist

$$
\operatorname{div} j\left(s_{1}, s_{2}\right)=\sum_{\nu=1}^{n}\left\langle h^{* v}, \nabla_{h_{v}} \cdot j\left(s_{1}, s_{2}\right)\right\rangle
$$

Aus (1) folgt:

$$
\begin{gathered}
\left\langle h^{* v}, \nabla_{h_{v}} \cdot j\left(s_{1}, s_{2}\right)\right\rangle=h_{v} \cdot\left\langle h^{* v}, j\left(s_{1}, s_{2}\right)\right\rangle-\left\langle\nabla_{h_{v}} \cdot h^{* v}, j\left(s_{1}, s_{2}\right)\right\rangle \\
=h_{v} \cdot H\left(\left(\Phi h^{* v}\right) s_{1}, s_{2}\right)-H\left(\left(\Phi\left(\nabla_{h_{v}} \cdot h^{*}\right)\right) s_{1}, s_{2}\right) .
\end{gathered}
$$

Die Gleichungen (2.9.1) und 4.1.1 implizieren nun

$$
\left\langle h^{* v}, \nabla_{h_{v}} \cdot j\left(s_{1}, s_{2}\right)\right\rangle=H\left(\left(\Phi h^{* v}\right)\left(\nabla_{h_{\nu}}^{\prime} \cdot s_{1}\right), s_{2}\right)+H\left(\left(\Phi h^{* v}\right) s_{1}, \nabla_{h_{v}}^{\prime} \cdot s_{2}\right) \text {. }
$$

Berüchsichtigt man noch (3) und die Definition von $D((1)$ in 4.7$)$ so erhält man

$$
\operatorname{div} j\left(s_{1}, s_{2}\right)=H\left(D s_{1}, s_{2}\right)-H\left(s_{1}, D s_{2}\right) .
$$

In Anlehnung an die Physik könnte man $j\left(s_{1}, s_{2}\right)$ das von $s_{1}$ und $s_{2}$ bestimmte Strömungsfeld nennen. Offensichtlich ist $\operatorname{div} j\left(s_{1}, s_{2}\right)=0$, wenn sowohl $D s_{1}=0$ als auch $D s_{2}=0$ ist.

Im weitern sei nun die Riemannsche Mannigfaltigkeit $R$ kompakt. Ist $e_{\Omega}$ das Volumelement von $R,[R]$ der Fundamentalzyklus von $R$, dann setzen wir für eine Funktion $\lambda \in \mathcal{F}_{C}$ :

$$
\int_{R} \lambda:=\left(\lambda e_{\Omega}\right)[R] .
$$

Auf $\Gamma \eta$ wird dann ein Skalarprodukt

$$
\left(s_{1}, s_{2}\right):=\int_{R} H\left(s_{1}, s_{2}\right)
$$

eingeführt, und aus (4) folgt dann

$$
\left(D s_{1}, s_{2}\right)-\left(s_{1}, D s_{2}\right)=0 .
$$

Also ist der Diracoperator formal selbstadjungiert.

4.9. $d+\delta$ als Diracoperator. Wir wollen in zwei bekannten Beispielen die Größen einer S-Struktur explizit angeben. Als erstes betrachten 
wir eine orientierte Riemannsche Mannigfaltigkeit $R=(M, Q)$ mit kovariantem Tangentialbündel $\left(\tau^{*}, Q\right)$; $d$ sei der Operator der äußeren Ableitung auf dem Bündel $\wedge \tau^{*}$ aller reellwertigen Differentialformen auf $M, \delta$ der zu $d$ adjungierte Operator ([12], §25, S. 125).

Wir erinnern an Beispiel 2 in 3.2: $\wedge \tau^{*}$ ist Spinorbündel einer natürlichen S-Struktur $\left(\wedge \tau^{*}\right)_{\tilde{\mu}}$ auf $R$. Dabei ist $\tilde{\mu}$, das das CliffordBündel von $\left(\tau^{*},-Q\right)$ auf $\wedge \tau^{*}$ operieren läßt, gemäß 2.6 durch eine Abbildung $v: \tau^{*} \rightarrow$ End $\wedge \tau^{*}$ erzeugt, die die Eigenschaft

$$
\left(v h^{*}\right) \omega=h^{*} \wedge \omega-\partial_{h^{*}} \omega, \quad \omega \in \Gamma \wedge \tau^{*}, h^{*} \in \varkappa^{*},
$$

hat: $\partial_{h^{*}}$ ist diejenige Antiderivation in $\Lambda \tau^{*}$, die die Werte

$$
\partial_{h^{*}} k^{*}=B\left(h^{*}, k^{*}\right), \quad h^{*}, k^{*} \in \mathscr{X}^{*}=\Gamma \tau^{*},
$$

annimmt, urd $B$ ist die zu $Q$ assoziierte Bilinearform auf $\tau^{*}$.

Diese S-Struktur wird nun vervollständigt, vorerst durch Einführung einer nichtentarteten symmetrischen Bilinearform $H$ auf $\left(\wedge \tau^{*}\right)_{\tilde{\mu}}$. Man verifiziert sofort, daß die natürliche Erweiterung $B^{\prime}$ von $B$ auf $<\tau^{*}$ ein solches $H$ ist. $B^{\prime}$ ist nämlich durch die Relationen definiert:

$$
\begin{gathered}
B^{\prime}=B \text { auf } \tau^{*} ; \\
B^{\prime}\left(h^{*} \wedge \omega, \omega^{\prime}\right)=B^{\prime}\left(\omega, \partial_{h^{*}}\left(\omega^{\prime}\right)\right.
\end{gathered}
$$

für alle $h^{*} \in \mathcal{C}^{*}$ und $\omega,()^{\prime} \in \Gamma \wedge \tau^{*}$.

Daraus folgt dann mit (1) die Beziehung

$$
B^{\prime}\left(\left(\tilde{\mu} h^{*}\right) \omega, \omega^{\prime}\right)=-B^{\prime}\left(\omega,\left(\tilde{\mu} h^{*}\right) \omega^{\prime}\right) .
$$

Also ist $B^{\prime}$ eine nichtentartete symmetrische Bilinearform auf $\left(\wedge \tau^{*}\right)_{\tilde{\mu}}$ (A und $\mathrm{B}$ in 4.1).

Weiter sei $\nabla$ der Levi-Cività-Zusammenhang auf $\left(\tau^{*},-Q\right)$, dessen Erweiterung auf $\wedge \tau^{*}$ wieder mit $\nabla$ bezeichnet sei (vgl. 2.9). Aus (2.9.3) folgt nun

$$
\nabla_{h} \cdot\left(\left(v h^{*}\right) \omega\right)=v\left(\nabla_{h} \cdot h^{*}\right) \omega+\left(v h^{*}\right)\left(\nabla_{h} \cdot \omega\right),
$$

somit ist gemäß 4.1.1 $\nabla$ ein linearer Zusammenhang auf $\left(\wedge \tau^{*}\right)_{\tilde{\pi}}$. Da $B$ und $\nabla$ die Ricci-Identität (2.9.1) erfüllen, tun dies auch $B^{\prime}$ und $\nabla$. Also ist $\left(\left(\wedge \tau^{*}\right), \nabla, B^{\prime}\right)$ eine vollständige S-Struktur auf $R$ (vgl. 4.5).

Der dazugehörige Diracoperator $D$ ist lokal durch die Gleichung (1) in 4.7 gegeben:

$$
D \omega=\sum_{v=1}^{n}\left(\tilde{\mu} h^{* v}\right)\left(\nabla h_{v} \cdot \omega\right), \quad n=\operatorname{dim} R,
$$

was sich mit (1) auch als 


$$
D \omega=\sum_{\nu=1}^{n}\left(h^{* \nu} \wedge \nabla_{h_{\nu}} \cdot \omega-\partial_{h^{* \nu}} \nabla_{h_{\nu}} \cdot \omega\right)
$$

schreiben läßt. Man verifiziert noch, daß für jede Differentialform $\omega$ auf $M$ gilt:

$$
\begin{aligned}
& d \omega=\sum_{\nu=1}^{n} h^{* v} \wedge \nabla_{h_{v}} \cdot \omega, \\
& \delta \omega=-\sum_{\nu=1}^{n} \partial_{h^{* v}} \nabla_{\boldsymbol{h}_{v}} \cdot \omega_{h_{\nu}} .
\end{aligned}
$$

Dabei ist es wesentlich, daß die Torsion von $\nabla$ verschwindet. Somit ist $d+\delta$ der Diracoperator der natürlichen S-Struktur $\left(\left(\wedge \tau^{*}\right) \tilde{\mu}, \nabla, B^{\prime}\right)$ auf $R$.

4.10. Kählersche Mannigfaltigkeiten. Als zweites Beispiel geben wir eine natürliche SC-Struktur auf einer Kählerschen Mannigfaltigkeit. Von einer Kählerschen Mannigfaltigkeit benützen wir folgende Eigenschaften (siehe [13] und [11], 114, théorème, S. 248): Sie ist eine Riemannsche Mannigfaltigkeit $K=(M, Q)$ zusammen mit einer Abbildung $J$ des Tangentialbündels $\tau$ von $M$ in sich, die den Bedingungen genügt:

$$
J^{2}=-\mathrm{id}_{\tau}
$$

$$
Q(J h)=Q(h) \text { für alle } h \in \mathcal{X},
$$

$$
\nabla \cdot J=0, \quad \nabla=\text { Levi-Cività-Zusammenhang auf }(M, Q) .
$$

Diese Gleichungen gelten auch, wenn $J$ durch die duale Abbildung $J^{*}: \tau^{*} \rightarrow \tau^{*}$ und $h$ durch $h^{*}$ ersetzt wird.

$\wedge \tau^{*}$ sei wie in 4.9. Das Bündel der komplexwertigen Differentialformen auf $M$, d.i. die Komplexifizierung von $\wedge \tau^{*}$, sei mit $\wedge \tau_{C}^{*}$ bezeichnet. Ist $\tau_{C}^{*}$ das Bündel der komplexwertigen 1-Formen auf $M$, dann ist $\wedge \tau_{C}^{*}=\wedge\left(\tau_{C}^{*}\right)$.

Es sei nun $\tau^{\prime}$ das Bündel derjenigen komplexwertigen 1 -Formen auf $M$ mit der Eigenschaft

$$
J^{*} h^{\prime}=i h^{\prime}, \quad i=\sqrt{-1} ;
$$

ebenso $\tau^{\prime \prime}$ das Bündel der komplexwertigen 1 -Formen $h^{\prime \prime}$ mit

$$
J^{*} h^{\prime \prime}=-i h^{\prime \prime}
$$

Es gilt

$$
\tau_{C}^{*}=\tau^{\prime} \oplus \tau^{\prime \prime}
$$


Wir zejgen vorerst, daß das äußere Bündel $\wedge \tau^{\prime}$ Spinorbündel einer SC-Struktur auf $K$ ist. Dazu genügt es, eine Abbildung $\varphi: \tau^{*} \rightarrow$ End $\wedge \tau^{\prime}$ mit der Eigenschaft

$$
\left(\varphi h^{*}\right)^{2}=-Q\left(h^{*}\right) \mathrm{id}
$$

$\mathrm{zu}$ konstruieren (SC 1 und SC 2 in 3.3; $Q$ in SC 2 ist wegen der Definition von S-Strukturen auf Riemannschen Mannigfaltigkeiten durch $-Q$ zu ersetzen, SC 1 ist erfüllt, wie man durch Dimensionsvergleich feststellt).

$\partial_{h^{*}}$ sei die in 4.9 eingeführte Antiderivation in $\wedge \tau^{*}$. Sie läßt sich zu einer Antiderivation $\partial_{h^{*}}$ in $\Lambda \tau_{C}^{*}$ erweitern. Da $\partial_{h^{*}}$ homogen vom Grad -1 ist, läßt es $\Lambda \tau^{\prime}$ invariant; wir setzen

$$
\partial_{h}^{\prime}:=\partial_{h^{*}} \mid \wedge \tau^{\prime}
$$

Weiter sei $P_{1,0}$ die Projektion $\tau_{C}^{*} \rightarrow \tau^{\prime}$. Dann definieren wir $\varphi h^{*}$ für $h^{*} \in \mathcal{C}^{*}=\Gamma \tau^{*}$ durch

$$
\left(\varphi h^{*}\right) \omega^{\prime}=\sqrt{2}\left[\left(P_{1,0} h^{*}\right) \wedge \omega^{\prime}-\partial_{h^{*}}^{\prime} \omega^{\prime}\right], \quad \omega^{\prime} \in \Gamma \wedge \tau^{\prime} .
$$

Mit (1) und (2), sowie mit (2) in 4.9 und der expliziten Darstellung

$$
P_{1,0} h^{*}=\frac{1}{2}\left(h^{*}-i J^{*} h^{*}\right)
$$

erhält man nun (4). Damit haben wir eine natürliche SC-Struktur $\left(\wedge \tau^{\prime}\right)_{\Phi}$ auf der Kählerschen Mannigfaltigkeit $K$ angegeben. Man bemerkt übrigens, daß die Forderung (3) nicht benützt wurde; $\left(\Lambda \tau^{\prime}\right)_{\Phi}$ hängt nur von der der Mannigfaltigkeit $K$ zugrundeliegenden Hermiteschen Struktur ab.

Nun zur Einführung einer nichtentarteten Hermiteschen Form $H$ auf $\left(\wedge \tau^{\prime}\right)_{\Phi}$. Es sei $B$ die zu $Q$ assoziierte Bilinearform auf $\tau^{*}$ und $B^{\prime}$ deren Erweiterung auf $\wedge \tau^{*}$ (vgl. 4.9). $B^{\prime}$ läßt sich zu einer Hermiteschen Form $H^{\prime}$ auf $\wedge \tau_{G}^{*}$ erweitern, deren Restriktion auf $\wedge \tau^{\prime}$ mit $H$ bezeichnet sei. Man verifiziert sofort, daß $H$ nichtentartet ist, und daß gilt:

$$
H\left(\left(\varphi h^{*}\right) \omega_{1}^{\prime}, \omega_{2}^{\prime}\right)=-H\left(\omega_{1}^{\prime},\left(\varphi h^{*}\right) \omega_{2}^{\prime}\right)
$$

$\omega_{1}^{\prime}, \omega_{2}^{\prime} \in \Gamma \wedge \tau^{\prime}, h^{*} \in \mathcal{X} *$

Für das folgende ist nun die Eigenschaft (3) wesentlich. Aus (7) folgt vorerst

$$
\nabla_{h} \cdot\left(P_{1,0} h^{*}\right)=P_{1,0}\left(\nabla \cdot h^{*}\right) .
$$

Also läßt die Erweiterung $\nabla$ des Levi-Cività-Zusammenhanges auf $\wedge \tau_{C}^{*}$ das Bündel $\wedge \tau^{\prime}$ invariant; wir setzen 
(9)

$$
\nabla^{\prime}:=\nabla \mid \wedge \tau^{\prime}
$$

und behaupten:

4.10.1. $\nabla^{\prime}$ ist ein linearer Zusammenhang auf $\left(\wedge \tau^{\prime}\right)_{\Phi} \cdot H$ und $\nabla^{\prime}$ erfüllen die Ricci-Identität 2.9.1.

Zum ersten Teil von 4.10.1: Für $\omega^{\prime} \in \Gamma \wedge \tau^{\prime}, h \in \mathcal{X}$ und $h^{*} \in \mathcal{X}^{*}$ ist

$$
\left(\Phi h^{*}\right) \omega^{\prime}=\left(\varphi h^{*}\right) \omega^{\prime}=\sqrt{2}\left[P_{1,0} h^{*} \wedge \omega^{\prime}-\partial_{h^{*}} \omega^{\prime}\right]
$$

also

$$
\nabla_{h}^{\prime} \cdot\left[\left(\Phi h^{*}\right) \omega^{\prime}\right]=\sqrt{2}\left[\nabla_{h}^{\prime} \cdot\left(P_{1,0} h^{*}\right) \wedge \omega^{\prime}+P_{1,0} h^{*} \wedge \nabla_{h}^{\prime} \cdot \omega^{\prime}-\nabla_{h} \cdot \partial_{h *}\left(\omega^{\prime}\right]\right.
$$

Wegen $\nabla_{h}^{\prime} \cdot \omega^{\prime}=\nabla_{h} \cdot \omega^{\prime}$ und (8) gilt

$$
\nabla_{h}^{\prime} \cdot\left(P_{1,0} h^{*}\right)=P_{1,0}\left(\nabla_{h} \cdot h^{*}\right) .
$$

Wegen 2.9.3 gilt

$$
\nabla_{h}^{\prime} \cdot \partial_{h^{*}} \omega^{\prime}=\nabla_{h} \cdot \partial_{h^{*}} \omega^{\prime}=\partial_{h^{*}} \nabla_{h} \cdot \omega^{\prime}+\partial_{\nabla_{h} \cdot h^{*}} \omega^{\prime},
$$

also ist

$$
\nabla_{h}^{\prime} \cdot\left[\left(\Phi h^{*}\right) \omega^{\prime}\right]
$$

$=\sqrt{2}\left[P_{1,0}\left(\nabla_{h} \cdot h^{*}\right) \wedge\left(\omega^{\prime}+P_{1,0} h^{*} \wedge \nabla_{h} \cdot \omega^{\prime}-\partial_{h^{*}} \nabla_{h} \cdot \omega^{\prime}-\partial_{\nabla_{h^{h}} \omega^{\prime}} \omega^{\prime}\right]\right.$.

Andererseits ist

$$
\Phi\left(\nabla_{h} \cdot h^{*}\right) \omega^{\prime}=\sqrt{2}\left[P_{1,0}\left(\nabla_{h} \cdot h^{*}\right) \wedge \omega^{\prime}-\partial_{\nabla_{h} \cdot h^{*}} \omega^{\prime}\right]
$$

und

$$
\left(\Phi h^{*}\right)\left(\nabla_{h}^{\prime} \cdot\left(\omega^{\prime}\right)=\sqrt{2}\left[P _ { 1 , 0 } h ^ { * } \wedge \nabla _ { h } \cdot \left(\omega^{\prime}-\partial_{h^{*}} \nabla_{h} \cdot\left(\omega^{\prime}\right] .\right.\right.\right.
$$

Es folgt die Gleichung 4.1.1: $(10)=(11)+(12)$, und $\nabla^{\prime}$ ist damit als linearer Zusammenhang auf $\left(\nabla \tau^{\prime}\right)_{\Phi}$ erwiesen.

Zum zweiten Teil von 4.10.1: $\nabla$ und $B$ erfüllen nach Definition die Ricci-Identität 2.9.1, also auch $\nabla$ und die Erweiterungen $B^{\prime}$ und $H^{\prime}$, also auch die Restriktionen $\nabla^{\prime}$ und $H$ auf $\wedge \tau^{\prime}$. Die Größen $\left(\wedge \tau^{\prime}\right)_{\Phi}, \nabla^{\prime}, H$ bilden also eine vollständige SC-Struktur auf $K$.

Der Diracoperator dieser SC-Struktur hat lokal die Form (1) von 4.7:

$$
D \omega^{\prime}=\sqrt{2} \sum_{\nu=1}^{n}\left[P_{1,0} h^{* \nu} \wedge \nabla_{h_{\nu}}^{\prime} \cdot \omega^{\prime}-\partial_{h^{* \nu}}^{\prime} \nabla_{h_{\nu}}^{\prime} \cdot \omega^{\prime}\right), \quad n=\operatorname{dim} K .
$$

Nach (4) in 9.1 gilt für die äußere Ableitung $d$ :

$$
d \omega^{\prime}=\sum_{\nu=1}^{n} h^{* \nu} \wedge \nabla_{h_{v}} \cdot\left(\omega^{\prime} .\right.
$$


Es sei nun $p^{\prime}: \wedge \tau_{C}^{*} \rightarrow \wedge \tau^{\prime}$ der Algebrahomomorphismus, der die Projektion $P_{1,0}: \tau_{C}^{*} \rightarrow \tau^{\prime}$ erweitert. Aus $\nabla_{h}^{\prime} \cdot()^{\prime}=\nabla_{h} \cdot \omega^{\prime}$ und $p^{\prime} \nabla_{h} \cdot \omega^{\prime}=\nabla_{h} \cdot \omega^{\prime}$ folgt dann

$$
p^{\prime} d \omega^{\prime}=\sum_{\nu=1}^{n} P_{1,0} h^{* \nu} \wedge \nabla_{h_{\nu}}^{\prime} \cdot \omega^{\prime} .
$$

$p^{\prime} d$ stimmt mit $d^{\prime}$ auf $\wedge \tau^{\prime}$ überein (für die Definition von $d^{\prime}$, sowie $\delta^{\prime}$, siehe [10], S. 34 und S. 43):

$$
d^{\prime} \omega^{\prime}=\sum_{\nu=1}^{n} P_{1,0} h^{* \nu} \wedge \nabla_{h_{v}}^{\prime} \cdot \omega^{\prime} .
$$

Der zu $d$ adjungierte Operator $\delta$ läßt $\wedge \tau^{\prime}$ invariant; denn dies gilt sowohl für die Antiderivationen $\partial_{h^{*}}$ als auch für $\nabla$, somit wegen (5) in 4.9, auch für $\delta$. Außerdem stimmt $\delta$ mit $\delta^{\prime}$ auf $\wedge \tau^{\prime}$ überein ([10], lcc. cit.). Also ist

$$
\delta^{\prime} \omega^{\prime}=-\sum_{v=1}^{n} \partial_{h^{* v}}^{\prime} \nabla_{h_{\nu}}^{\prime} \cdot \omega^{\prime} .
$$

Insgesamt folgt dann aus (13), (14) und (15), daß der Diracoperator $D$ der natürlichen SC-Struktur $\left(\wedge \tau^{\prime}\right)_{\Phi}$ auf der Kählerschen Mannigfaltigkeit $K$ bis auf einen Faktor mit $d^{\prime}+\delta^{\prime}$ übereinstimmt:

$$
D=\sqrt{2}\left(d^{\prime}+\delta^{\prime}\right) .
$$

Universität Zürich

Mathematisches Institut

CH-8032 Zürich

Schweiz 


\section{Literatur}

[1] Atiyah, M. F., und I. M. Singer: The index of elliptic operators on compact manifolds. - Bull. Amer. Math. Soc. 69, 1963, S. 422-433.

[2] BAss, H.: Algebraic $K$-theory. - Mathematics Lecture Note Series. W. A. Benjamin, Inc., New York/Amsterdam, 1968.

[3] Chevalley, C.: The algebraic theory of spinors. - Columbia Bicentennial Editions and Studies. Columbia University Press, Morningside Heights (N.Y.), 1954.

[4] -»- The construction and study of certain important algebras. - Publications of the Mathematical Society of Japan 1. The Mathematical Society of Japan, Tokyo, 1955.

[5] Donovan, P., und M. Karoubi: Graded Brauer groups and $K$-theory with local coefficients. - Inst. Hautes Études Sci. Publ. Math. 38, 1970, S. $5-2.5$.

[6] Frenkel, J.: Cohomologie à valeurs dans un faisceau non abélien. - C. R. Acad. Sci. Paris 240, 1955, S. 2368-2370.

[7] FröHLICH, A.: Hermitian and quadratic forms over rings with involution. Quart. J. Math. Oxford Ser. (2) 20, 1969, S. 297-317.

[8] Haefliger, A.: Sur l'extension du groupe structurale d'un espace fibré. C. R. Acad. Sci. Paris 243, 1956, S. 558-560.

[9] Hirzebruch, F.: Topological methods in algebraic geometry. - [Third enlarged edition.] Die Grundlehren der mathematischen Wissenschaften 131. Springer-Verlag, Berlin / Heidelberg / New York, 1966.

[10] Lichnerowicz, A.: Théorie globale des connexions et des groupes d'holonomie. - Consiglio Nazionale delle Ricerche, Monografie Matematiche 2. Edizioni Cremonese, Roma, 1957.

[11] DE Rhanr, G.: Variétés différentiables. Formes, courants, formes harmoniques. - Publications de l'Institut de Mathématique de l'Ćniversité de Nan, cago III. Actualités Sci. Indust. 1222. Hermann \& Cie, ÉditeursParis, 1955.

[12] WEIL, A.: Introduction à l'étude des variétés kählériennes. - Publications de l'Institut de Mathématique de l'Université de Nancago VI. Actualités Sci. Indust. 1267. Hermann, Paris, 1957. 